\title{
Desempeño en estocástica entre profesores de educación secundaria: un estudio exploratorio en dos regiones de Costa Rica y México
}

\author{
Performance in Stochastic between Secondary School Teachers: \\ An Exploratory Study in Two Regions of Costa Rica and Mexico
}

\author{
Humberto Cuevas ${ }^{1}$ \\ Greivin Ramírez ${ }^{2}$
}

\begin{abstract}
Resumen Se presentan los resultados de un estudio exploratorio sobre el desempeño en tópicos estocásticos elementales de 111 profesores de educación secundaria de México y Costa Rica. Se elaboró y validó un instrumento de 16 ítems, de acuerdo con los lineamientos del Programa Internacional de Evaluación de los Alumnos (PISA). Los resultados indican que el desempeño alcanzado no es suficiente para cumplir con los estándares educativos establecidos en los programas de estudio. Fue detectado que más de la mitad de profesores (59) desconocen los principios básicos de la probabilidad, 68 tienen dificultades para interpretar datos agrupados en gráficas y tablas, 68 no clasifican variables de forma precisa, además de la dificultad para diferenciar entre fenómenos aleatorios y determinísticos (19). Se infiere que en la mayor parte de los tópicos el dominio disciplinar fue similar en ambas naciones, mostrando diferencias a favor de los costarricenses en la clasificación de variables y a favor de los mexicanos en la independencia de eventos. Se recomienda a las instituciones considerar en sus programas el desarrollo histórico de la
\end{abstract}

Fecha de recepción: 2 de febrero de 2017. Fecha de aceptación: 17 de julio de 2017

1 Instituto Tecnológico de Chihuahua II, Departamento de Ciencias Básicas, humberto.cuevas@itchihuahuaii.edu.mx.

2 Instituto Tecnológico de Costa Rica, Escuela de Matemática, gramirez@itcr.ac.cr. 
probabilidad y la estadística, el uso del lenguaje propio de estas disciplinas, analizar información en distintas representaciones, así como resolver problemas dentro y fuera del ámbito escolar.

Palabras clave: educación estadística; estándares curriculares; formación de profesores; prueba PISA; educación secundaria.

\begin{abstract}
The results of an exploratory study on the performance in elementary stochastic variables of 111 secondary school teachers from Mexico and Costa Rica are shown. An instrument of 16 items was developed and validated according to the guidelines of the Program for International Student Assessment (PISA). The findings indicate that the performance is not sufficient to comply with the stochastic educational standards established in study programs. It was determined that more than half of teachers (59) do not know basic probability principles, 68 have difficulties interpreting grouped data in graphs and tables, 68 do not classify variables accurately, and there are also difficulties differentiating between random and deterministic phenomena (19). It is inferred that in most of the topics the disciplinary domain was similar in both countries, showing differences in favor of the Costa Ricans in variable classification and in favor of the Mexicans in event independence. It is recommended that institutions consider in their programs the historical development of probability and statistics, use the discipline's language, and analyze information in different representations, as well as solve problems inside and outside the school environment.
\end{abstract}

Keywords: Statistical education; curricular standards; teacher training; PISA test; secondary education.

\title{
1. INTRODUCCIÓN
}

A partir de las últimas décadas del siglo Xx se ha promovido el desarrollo de una alfabetización estocástica en todos los estratos sociales, considerando la institución escolar y al profesorado como ejes indispensables en el logro de este propósito. Este proceso de alfabetización ha transitado por diversas etapas, una de las más significativas fue la discusión sobre la situación de la estocástica 
como disciplina científica, en la que se examinó su uso como instrumento de apoyo para la investigación y se ponderó el grado de dependencia con la matemática como área integradora.

También se ha incentivado el establecimiento de estándares educativos en varios campos de conocimiento, especialmente en ciencia, lenguaje y matemáticas. La aplicación y valoración de pruebas estandarizadas constituye el mecanismo principal que se ha utilizado para introducir estos estándares; en consonancia con lo anterior, se han incluido más tópicos estocásticos en el currículo escolar de educación primaria, secundaria y bachillerato en naciones de los cinco continentes, destacando las integrantes de América Latina: Chile, Argentina, Brasil, México, Costa Rica, Colombia y Venezuela.

\subsection{ANTECEDENTES}

Costa Rica y México destacan por la magnitud de los ajustes curriculares efectuados y su interés en establecer programas de apoyo académico al profesorado que debe enseñar estocástica en estos niveles educativos. Ambas naciones también han promovido el establecimiento de estándares internacionales en sus currículos, especialmente los elaborados por el National Council of Teachers of Mathematics (NCTM) y los formulados por la Organisation for Economic Cooperation and Development (OECD) evaluados en su Program for International Student Assessment (PISA).

En 2012 el Ministerio de Educación Pública (MEP) de Costa Rica efectuó ajustes en el currículo escolar, y en el área matemáticas el nuevo programa de estudios se organizó en cinco áreas, a saber: números, geometría, medidas, relaciones y álgebra, además de probabilidad y estadística (MEP, 2012). Entre los ajustes más representativos destaca el espacio concedido a los tópicos estadísticos del primero al duodécimo grado, aproximadamente $20 \%$ del total de contenidos en los seis niveles iniciales, y alcanzando 30\% en los últimos seis.

De igual manera, en México se han efectuado adecuaciones al currículo en los niveles educativos primarios; desde la Reforma Integral de la Educación Básica (RIEB), promovida por la Secretaría de Educación Pública (SEP) en 2011, se observaron cambios; la estructura curricular fue organizada en cuatro campos de formación, y en matemáticas establecieron estándares curriculares en cuatro áreas claramente delimitadas: sentido numérico y pensamiento 
algebraico; forma, espacio y medida; manejo de información, y actitud hacia el estudio de las matemáticas. En los primeros seis grados -educación primaria-, los programas de estudio fueron estructurados en cinco bloques que plantean situaciones de complejidad ascendente. Cada bloque incluye contenidos de cada campo, como mecanismo para que el estudiante tenga una visión holística de las matemáticas. Aproximadamente 25\% de los temas tienen relación directa con el manejo de la información. Respecto al séptimo, octavo y noveno grados -educación secundaria-, la estructura por bloques y el porcentaje asignado es similar. No obstante, es hasta el duodécimo grado de bachillerato donde se incluye un curso de probabilidad y estadística de cinco horas semanales.

Es necesario señalar que la educación secundaria costarricense está programada entre el séptimo y duodécimo grado, y el rango de edades de los estudiantes oscila entre los 13 y 17 años. En el caso de México, la educación secundaria está ubicada entre el séptimo y noveno grado, y las edades de los alumnos fluctúan entre los 12 y 14 años. En la Figura siguiente se muestra la estructura global de ambos sistemas educativos.

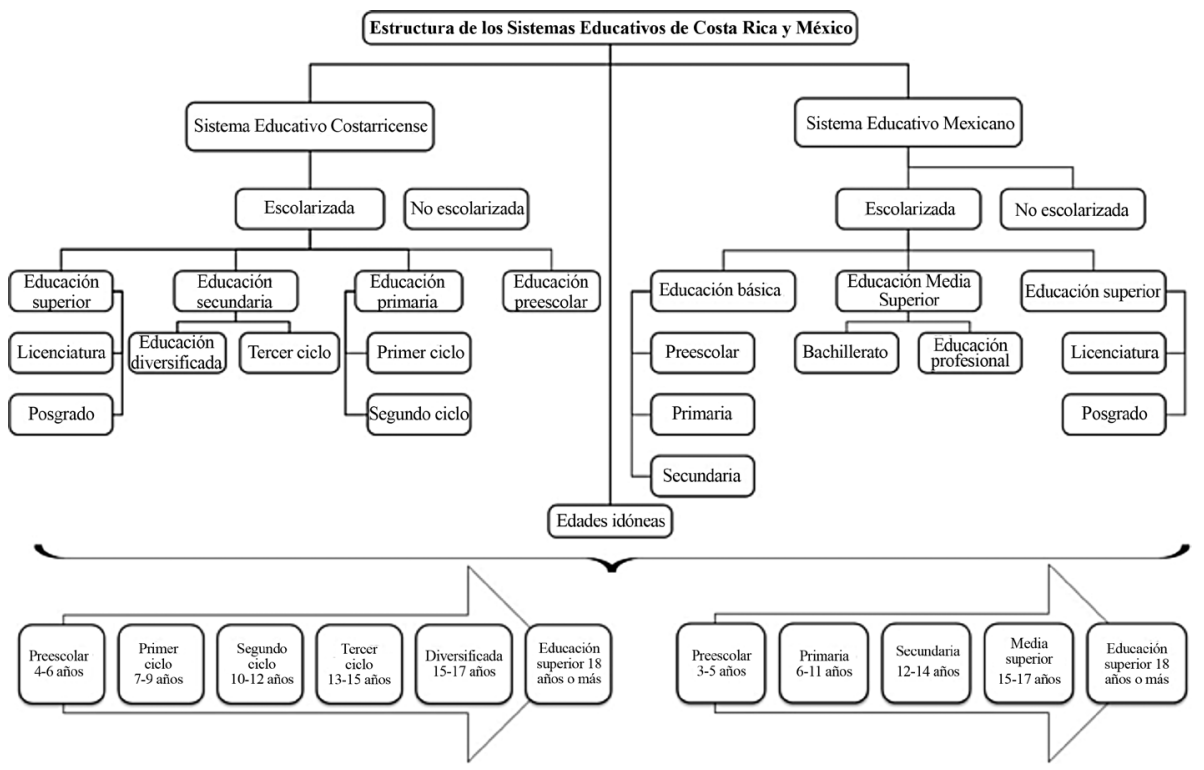

Figura 1. Estructura general de los sistemas educativos de Costa Rica y México. 


\subsection{ESTUDIOS REPRESENTATIVOS}

De manera paralela, se han multiplicado los estudios que examinan la evolución de la estadística y la probabilidad en el ámbito escolar, especialmente en lo relacionado con el logro de aprendizajes, prácticas de enseñanza, actitudes y saberes del profesorado sobre ellas.

Shaughnessy (2007) hizo un análisis de la evolución de la educación estadística en los últimos años y destacó características de estudios sobre el aprendizaje de esta disciplina realizados por académicos estadounidenses y neozelandeses; Watson (2013) analizó tópicos relacionados con la alfabetización estadística y el razonamiento, además de presentar actividades útiles para que el profesorado las evalúe en el aula de clase; por otra parte, Marz y Kelchtermans (2013) indagaron en la complejidad de implementar procesos de innovación en el ámbito educativo y mostraron los resultados de un estudio exploratorio acerca de las percepciones del profesorado respecto a los nuevos planes y programas de trabajo en la región Flamenca de Bélgica.

En el estudio de Zieffler, Park, Garfield, DelMas y Bjornsdottir (2012) se diseñó un instrumento de 50 ítems para evaluar las actitudes, prácticas de enseñanza y evaluación de profesores de cursos introductorios de estadística; aplicaron el instrumento a 101 participantes que enseñaban esa materia en diversas áreas como Artes, Educación, Psicología, Ingeniería y Biología, entre otras, mientras que Biehler (2016) describe las actividades de un curso-piloto para profesores que deben enseñar tópicos estocásticos en educación secundaria en Alemania, señalando la importancia de las herramientas digitales y la incorporación de la simulación en la enseñanza.

En el ámbito iberoamericano también se han efectuado estudios representativos. Batanero, Arteaga y Contreras (2011) hicieron una reflexión acuciosa sobre los retos que enfrenta la educación estadística en España, debido a las nuevas directrices curriculares en la enseñanza obligatoria, y presentaron recomendaciones metodológicas con el fin de promover el desarrollo del razonamiento estadístico.

En México algunas investigaciones, como la de Insunza y Guzmán (2011), examinaron la comprensión y conocimiento en probabilidad de profesores mexicanos de educación secundaria -séptimo, octavo y noveno grado- y encontraron que el nivel de razonamiento en problemas de combinatoria no es el esperado; también detectaron concepciones erróneas en tópicos importantes de esta área. Los autores plantean que esta es una problemática aguda, debido a la formación 
deficiente del profesorado en el campo de la probabilidad y a la orientación determinista de los programas de trabajo.

Huerta (2016) propone recientemente una alternativa para resolver problemas de probabilidad a través de la simulación, señalando las virtudes heurísticas que potencian la exploración y el descubrimiento constructivo; advierte, asimismo, las diferencias en la formación de profesores que ejercen la enseñanza en primaria y secundaria, sugiriendo que en los primeros es importante que el método se utilice para resolver problemas de probabilidad a partir de situaciones reales y transitar a un proceso de modelado trabajando con modelos específicos en un ir y venir en estos dos estadios para el caso de los segundos señala que el procedimiento debe ser el contrario, es decir, del modelo debe transitarse a la situación real.

Por su parte, Alsina y Vásquez (2016) analizaron los conocimientos probabilísticos de docentes chilenos y encontraron dificultades tanto en lo didáctico como en lo disciplinar. También en Chile, Estrella, Olfos y Mena-Lorca (2015) indagaron sobre los "saberes" de 85 profesores de educación primaria, de forma paralela a la de sus propios alumnos de cuarto y séptimo grado; el propósito fue construir un instrumento confiable y válido sobre el conocimiento pedagógico del contenido de estadística, al igual que examinar el desempeño de profesores y estudiantes.

Los resultados obtenidos en estos estudios evidencian el interés por examinar los retos de la incorporación de la estadística y la probabilidad en el currículo escolar, así como la preocupación por saber si el profesorado tiene el dominio disciplinar suficiente para enseñarlas en la medida que los estándares curriculares lo exigen.

\subsection{INTERROGANTES DE INVESTIGACIÓN}

En virtud del interés creciente por incorporar estos tópicos a los programas de estudio, en el establecimiento de estándares educativos internacionales y el reconocimiento de la importancia del profesorado en la enseñanza, es primordial dar respuesta a las siguientes interrogantes: ¿̇uál es el grado de dominio en tópicos de estocástica elemental de profesores de enseñanza secundaria?, ¿en qué medida dicho dominio es suficiente para cumplir con los estándares curriculares exigidos en los programas de estudio?, ¿cuáles son las principales áreas de mejora 0 necesidades de actualización que presenta el profesorado? y cexisten diferencias significativas en los conocimientos de los docentes en ambas naciones? 


\subsection{PROPÓSITO DEL ESTUDIO}

En este artículo se presentan los resultados de un estudio exploratorio de carácter comparativo entre Costa Rica y México, sobre el desempeño en tópicos estocásticos elementales de 111 profesores en activo que enseñaban matemáticas entre el séptimo y duodécimo grado, específicamente dentro del marco de los tópicos y criterios que se evalúan en la prueba PISA formulada por la OECD.

El estudio fue auspiciado por dos instituciones universitarias de prestigio reconocido, ambas dedicadas a la formación de profesionistas que, entre otras funciones, tienen como encomienda la formación académica en los campos de las matemáticas y estadística en diversos niveles educativos, entre ellos los examinados en este documento.

\section{MARCO DE REFERENCIA}

El Ministerio de Educación de Costa Rica y la Secretaría de Educación Pública de México han adoptado las políticas educativas emanadas de la OECD que han sido puestas en operación a través de distintos mecanismos, entre ellas la prueba PISA. Esta adopción ha ocasionado que las modificaciones al currículo sean acordes a las recomendaciones de este organismo multilateral y que, aun cuando no se explicite de manera directa, la calidad de sus sistemas educativos se mida en función de los puntajes que obtienen sus estudiantes en la prueba que se aplica cada trienio.

La OECD es probablemente el organismo multilateral que ha ejercido mayor influencia en el diseño, aplicación y seguimiento de pruebas estandarizadas para evaluar la calidad educativa. En 1997 creó y aplicó por primera vez la prueba PISA, cuyo objetivo principal es dar seguimiento a los resultados obtenidos en sus países miembros y en los que libremente se adhieren.

La prueba examina los dominios en lectura, matemáticas y ciencias. El dominio de matemáticas tiene cuatro sub-escalas, 1) Cantidad, 2) Espacio y Forma, 3) Cambio y Relaciones, y 4) Incertidumbre y datos; para cada una existen seis niveles de desempeño donde se indica qué deben ser capaces de hacer los estudiantes. En el último caso, se integra el tratamiento estadístico de los datos y su probabilidad de ocurrencia.

Los conceptos y actividades examinados incluyen la recolección, el análisis y organización de datos, además de su inferencia. En la Tabla 1 se describen las tareas y niveles de desempeño en esta sub-escala: 
Tabla 1. Descripción de las tareas y niveles de desempeño en incertidumbre y datos, PISA 2012.

\section{Niveles Los estudiantes deben poder:}

Interpretar, evaluar y reflexionar críticamente sobre una serie de datos estadísticos o probabilísticos, sobre información y situaciones complejas, para analizar los problemas. Saben aplicar conocimientos y razonamientos sujetos a varios elementos del problema; entienden las conexiones entre los datos y las situaciones que representan y son capaces de sacar provecho de estas conexiones para explorar plenamente las situaciones del problema. Llevan a cabo técnicas de cálculo apropiadas para explorar los datos o resolver problemas probabilísticos; y pueden producir y comunicar conclusiones, razonamientos y explicaciones.

Interpretar y analizar una serie de datos estadísticos o probabilísticos, información y situaciones, para resolver el problema en contextos complejos que requieren la vinculación de diferentes componentes del mismo. Pueden utilizar el razonamiento proporcional de forma eficaz para vincular los datos de una muestra a la población a la que representan; pueden interpretar las series de datos adecuadamente a lo largo del tiempo, y ser sistemáticos en el uso y la exploración de datos. Saben utilizar los conceptos estadísticos y probabilísticos para reflexionar, sacar conclusiones y generar y comunicar los resultados.

Elaborar y emplear las representaciones de una serie de datos y procesos estadísticos y probabilísticos para interpretar los datos, la información y las situaciones, con el fin de hallar la solución del problema. Pueden trabajar de modo eficaz con restricciones, tales como las condiciones estadísticas que pueden aplicar en un experimento de muestreo, y pueden interpretar y convertir los datos entre dos representaciones relacionadas (tales como un gráfico y una tabla de datos). Saben desarrollar el razonamiento estadístico y probabilístico para llegar a conclusiones contextuales.

Trabajar con datos e interpretar información estadística procedente de una sola representación que incluye múltiples fuentes de información (por ejemplo, un gráfico que representa varias variables) o procede de dos representaciones de datos relacionadas, tales como una tabla de datos simple y un gráfico. Pueden trabajar e interpretar los conceptos y convenciones estadísticas y probabilísticas descriptivas, como el lanzamiento de una moneda o la lotería, además de poder sacar conclusiones a partir de los datos, por ejemplo, calculando o utilizando las medidas simples del centro y dispersión. Son capaces de llevar a cabo un razonamiento básico de estadística y probabilidad en contextos sencillos. 
Identificar, extraer y comprender los datos estadísticos presentados en una forma simple y conocida, por ejemplo en forma de tabla, gráfico de barras o gráfico de segmentos. Pueden identificar, entender y utilizar los conceptos de estadística y probabilidad básica y descriptiva en contextos cotidianos, como el lanzamiento de una moneda o tirar los dados. En este nivel pueden interpretar los datos en representaciones simples y aplicar los procedimientos de cálculo apropiados que conecten los datos facilitados al contexto del problema.

Identificar y leer la información presentada en tablas pequeñas o gráficos simples y bien etiquetados para localizar y extraer los valores de datos específicos, ignorando la información distractora, e identificar cómo están relacionados con el contexto. Reconocer y utilizar los conceptos básicos de aleatoriedad para identificar ideas erróneas en conceptos empíricos conocidos, como los resultados de la lotería.

La prueba PISA tiene atributos que la hacen útil como marco de referencia para medir desempeños; entre los más representativos destaca la solidez en sus propiedades de medida y las múltiples aplicaciones y revisiones que se le han realizado para evaluar su confiabilidad y validez.

Barquín, Gallardo, Fernández, Yus, Sepúlveda y Serván (2011) señalan que el profesorado no prepara a sus alumnos para responder satisfactoriamente la prueba PISA; también sugieren que, debido al carácter internacional de PISA, se deberían tomar en cuenta otras variables culturales, sociales y económicas para tener una mejor lectura de sus resultados.

De igual manera, Pedró (2012) subraya el carácter prescriptivo de la prueba y cuestiona si se pueden explicar los resultados que obtienen los estudiantes de una nación sin que se considere el contexto del aula.

En el caso de Costa Rica y México, los resultados obtenidos por los alumnos en la prueba PISA no han sido satisfactorios, especialmente en el área de alfabetización matemática.

\section{MÉTODO}

La investigación es cuantitativa y se enmarca dentro de un estudio exploratorio. Forma parte de un estudio más amplio donde se caracterizó a esta muestra en tres grandes dimensiones: 1) Social - económica, 2) Pedagógica - didáctica actitudinal, y 3) Disciplinar, que es la examinada en este documento. 


\subsection{POBLACIÓN BAJO ESTUDIO}

La población bajo estudio consistió en profesores en activo de educación secundaria que enseñaban tópicos estocásticos entre el séptimo y duodécimo grado en Costa Rica, y entre primero de secundaria y tercero de bachillerato en México.

Es importante subrayar que existen diferencias en la formación académica de los participantes. En Costa Rica, por ejemplo, el profesorado se forma principalmente en instituciones públicas de educación superior como el Instituto Tecnológico de Costa Rica (ITCR), la Universidad de Costa Rica (UCR), Universidad Nacional (UNA), Universidad Estatal a Distancia (UNED) y la Universidad Técnica Nacional de Costa Rica (UTN). Así, el profesorado generalmente tiene el título de bachiller o licenciado en Enseñanza de la Matemática.

En México el sistema de formación de profesores es heterogéneo, debido especialmente a la diferenciación curricular para atender necesidades específicas y a los niveles y modalidades presentes en la educación básica; por tanto, existen instituciones formadoras para educación primaria, secundaria, educación física, educación tecnológica, tele-secundaria, artística, indígena, en educación e innovación pedagógica, entre otras. Las instituciones más destacadas son las Escuelas Normales (EN), Escuelas Normales Superiores (ENS) y Universidades Pedagógicas (UP).

\subsection{MUESTREO}

Se utilizó un muestreo no probabilístico para seleccionar a los participantes. Específicamente se usó un muestreo por selección intencionada de 111 profesores, 33 costarricenses de la provincia de Cartago y 78 mexicanos de la región centro-sur del estado de Chihuahua, $47 \%$ del sexo femenino y $53 \%$ del masculino.

La elección de este tipo de muestreo obedeció a varios motivos, a saber: 1) No se tuvo acceso a una base de datos completa y confiable del número de profesores adscritos a instituciones educativas en los grados de interés, es decir, no fue posible establecer con exactitud un marco muestral; 2) Se contó con docentes voluntarios que reunían el perfil deseado; 3) Se consideró pertinente examinar primero la existencia de patrones en los resultados del estudio exploratorio, antes de optar por una técnica de muestreo más onerosa en términos de tiempo y costo económico.

Existe gran similitud entre los atributos sociales de los profesores de la provincia de Cartago y la región centro-sur del estado de Chihuahua, ambos 
grupos contaban con los mismos servicios en sus viviendas y en proporciones similares (Internet, computadora, lavadora, televisor, refrigerador y servicio de televisión de paga, entre otros), con excepción de las categorías relacionadas con el tipo de vivienda (propia, rentada o prestada) y la propiedad o no de un automóvil, en las que hubo diferencias (los mexicanos contaban en su mayoría con vehículo, a diferencia de los costarricenses).

\subsection{INSTRUMENTOS UTILIZADOS PARA ACOPIAR Y TRATAR LA INFORMACIÓN}

Fue elaborada una prueba de dominio disciplinar (ver Anexo 1) bajo el criterio de juicio de expertos en matemáticas, estadística y educación matemática. La clasificación de los ítems se basó en los seis niveles de competencia-desempeño contenidos en la evaluación de la prueba PISA, y se estructuró a la luz de los currículos de educación estadística vigentes en Costa Rica y México (INEE, 2005).

La prueba de dominio disciplinar se elaboró y adaptó para explorar en qué medida los profesores daban respuestas acertadas a ítems equivalentes a los incluidos en la prueba que se aplica a sus estudiantes cada trienio. El idioma español es la lengua oficial en ambas naciones; no obstante, hay diferencias de dialecto que se tuvieron en cuenta al momento de construir, probar y validar cada ítem. Ambos aspectos, dominio disciplinar y lenguaje denotativo, no pueden disociarse en un instrumento de esta naturaleza.

En la siguiente tabla se presenta la clasificación de los ítems que integraron la prueba, en términos de su nivel en la escala que establece PISA y la temática examinada.

Tabla 2. Clasificación de ítems para examinar el dominio disciplinar del profesorado.

\begin{tabular}{ccl}
\hline Ítem & Nivel de escala de pisa & \multicolumn{1}{c}{ Temática } \\
\hline 1 & 4 & Variabilidad \\
2 & 1 & Evento \\
3 & 3 & Experimento aleatorio \\
4 & 3 & Interpretación gráfica \\
5 & 3 & Interpretación gráfica \\
6 & 2 & Medidas de tendencia central \\
7 & 4 & Tabla de frecuencias
\end{tabular}




\begin{tabular}{rll}
8 & 5 & Medidas de tendencia central \\
9 & 4 & Probabilidad con porcentajes \\
10 & 6 & Probabilidad condicional \\
11 & 2 & Medidas de tendencia central \\
12 & 3 & Clasificación de variables \\
13 & 3 & Clasificación de variables \\
14 & 3 & Clasificación de variables \\
15 & 5 & Diagrama de Golback \\
16 & 4 & Eventos independientes \\
\hline
\end{tabular}

\subsection{Protocolo DE tRabajo}

El proceso de construcción, medición de la confiabilidad y validez del instrumento consistió en cinco etapas claramente delimitadas.

\subsubsection{Construcción, confiabilidad y validación del instrumento}

a) El equipo de trabajo elaboró un banco de ítems teniendo como referencia los niveles de PISA señalados líneas atrás. Algunos fueron adaptados de investigaciones en educación estadística desarrolladas a nivel internacional, como las de Ballestero (2006); Garfield, delMas y Chance (2004) y MEP (2012). Estos ítems fueron evaluados, por criterio de expertos, en dos aspectos: 1) Claridad: que el ítem esté bien redactado en cuanto a los datos proporcionados y a lo que se solicita; 2) Pertinencia: que el ítem sea adecuado para evaluar conceptual y procedimentalmente el contenido al que pertenece.

b) Se construyeron dos pruebas piloto, A y B, cada una integrada por 13 ítems distribuidos según los niveles de PISA, como una campana de Gauss, a saber: un ítem del nivel 1, dos del nivel 2, tres del nivel 3, cuatro del nivel 4, dos del nivel 5 y un ítem del nivel 6 .

c) Con el objetivo de examinar la confiabilidad, se efectuó una prueba piloto con 50 profesores en activo de ambas naciones, que enseñaban tópicos estocásticos entre el séptimo y duodécimo grado. Específicamente, se utilizó el método de formas paralelas o coeficiente de equivalencia y estabilidad. 
d) Luego se realizaron los análisis técnicos de dificultad y discriminación de los ítems, además del estudio de confiabilidad de ambas pruebas.

e) Por último, fue elaborado un único cuestionario de 16 ítems, a partir de las pruebas piloto $\mathrm{A}$ y $\mathrm{B}$, distribuidos igualmente en forma de campana de Gauss según los niveles de PISA y que cumplieran con los parámetros aceptables de discriminación y dificultad de los ítems propuestos en los formularios piloto.

Con el propósito de evaluar la dificultad y discriminación de los ítems, se clasificaron los grupos en relación al que obtuvo la puntuación más alta y la más baja (33\%, 33\%), y para hacerlo, se utilizaron las calificaciones totales en la prueba. Habitualmente esta técnica es llamada "de colas", pues se espera que las calificaciones se distribuyan en forma normal. Enseguida se hizo un recuento del número de personas dentro de cada grupo que respondió correctamente el ítem.

Para el índice de dificultad fue calculada la suma entre el número de personas en el grupo de alta puntuación que respondieron correctamente el ítem, y el número de personas del grupo de baja puntuación que respondieron correctamente. Así, el índice de dificultad del ítem es la razón de la suma de respuestas correctas en ambos grupos y el número total de personas que consideran ambos grupos.

Respecto al índice de discriminación, se calculó una puntuación de diferencia entre el número de personas en el grupo de alta puntuación, que respondieron correctamente el ítem, y el número de personas del grupo de baja puntuación que respondieron también correctamente. En consecuencia, el índice de discriminación del ítem es la razón de la diferencia y el número de personas en el grupo de alta puntuación.

La interpretación de los valores de la dificultad se hizo de acuerdo con lo planteado por Meneses (1993) a los siguientes intervalos en los valores de medidas: 1) 1 a 0.85 son interpretados como muy fáciles (no se recomiendan, se desechan); 2) 0.85 a 0.65 como ítems fáciles; 3) 0.64 a 0.35 como normales; 4) 0.34 a 0.15 como difíciles; 5) 0.15 a 0 como extremadamente difíciles (no se recomiendan, se desechan). Este autor también señala que los ítems que discriminan deben presentar valores iguales o superiores a 0.30, de no ser así, deberán ser descartados. En la prueba definitiva se incorporaron ítems de dificultad fácil, normal y difícil, todos ellos discriminando.

Para determinar del grado de confiabilidad -consistencia interna- se calculó el coeficiente Alfa de Cronbach. La prueba A arrojó una confiabilidad de 0.651, 
mientras que la B fue de 0.625 , lo cual indica que ambas pruebas presentan confiabilidad aceptable, al ser tan pocos ítems por prueba.

\subsubsection{Aplicación}

Se suministró por escrito la prueba a cada participante. Se les indicó que el objetivo era obtener información sobre el grado de dominio en las áreas de probabilidad y estadística, señalando que no debían escribir su nombre, con el fin de tener confidencialidad total en las respuestas. La duración de la aplicación fue inferior a las dos horas y no hubo diferencias significativas en el tiempo utilizado entre los grupos.

Posteriormente los resultados obtenidos fueron registrados en una base de datos y se realizaron los análisis estadísticos descriptivos e inferenciales con el programa de cómputo SPSS V. 21.

\section{RESULTADOS Y SU DISCUSIÓN}

La prueba de dominio disciplinar examinó diversos tópicos estocásticos, la mayoría incluidos en los programas de estudio habituales entre el séptimo y duodécimo grado, en ambas naciones.

Para realizar el análisis, los ítems fueron separados por temáticas y se obtuvo el desempeño promedio total y por nación; además, se efectuaron pruebas de hipótesis de diferencia de proporciones de acierto, en las diversas temáticas, entre ambas naciones. Destaca sobremanera las dificultades de los profesores para interpretar tablas y gráficas, en los que solo $38.74 \%$ del total de participantes acertaron en promedio para los cinco ítems correspondientes a dicha temática.

Además, hubo una inadecuada clasificación de variables, pues solamente $39.04 \%$ acertó al realizarla, siendo un poco superior el rendimiento de los profesores en Costa Rica que los de México. La siguiente tabla presenta un resumen global de los resultados. 
Tabla 3. Rendimiento global y por nación de los participantes según temáticas.

\begin{tabular}{|c|c|c|c|c|}
\hline \multirow[b]{2}{*}{ Temática } & \multirow[b]{2}{*}{ Ítems } & \multirow{2}{*}{$\begin{array}{c}\% \text { de } \\
\text { acierto } \\
\text { total }\end{array}$} & \multicolumn{2}{|c|}{$\begin{array}{l}\% \text { de acierto } \\
\text { por nación }\end{array}$} \\
\hline & & & Costa Rica & México \\
\hline Interpretación de tablas y gráficos & $1,4,5,7,9$ & 38.74 & 40.00 & 38.21 \\
\hline Experimentos aleatorios y deterministas & 2 y 3 & 82.88 & 77.27 & 85.26 \\
\hline Medidas de tendencia central & 6,8 y 11 & 44.14 & 42.42 & 44.87 \\
\hline Probabilidad & 10 & 46.85 & 54.55 & 43.59 \\
\hline Clasificación de variables & 12,13 y 14 & 39.04 & 57.58 & 31.20 \\
\hline Diagrama de Golback & 15 & 74.77 & 66.67 & 78.21 \\
\hline Independencia de eventos & 16 & 79.28 & 63.64 & 85.90 \\
\hline
\end{tabular}

En virtud de que fueron examinadas siete temáticas con ítems de distinto grado de dificultad, se consideró necesario efectuar un análisis más detallado. A continuación se presentan los resultados obtenidos en cada uno.

\subsection{INTERPRETACIÓN DE TABLAS Y GRÁFICOS}

En el ítem 1 (nivel 4 según PISA) fue examinado el tópico variabilidad, presenta dos gráficas de barras con distinta distribución y se cuestionó cuál tenía mayor variabilidad. $21.62 \%$ de los participantes respondió correctamente "En la distribución A", y 71.2\% señaló erróneamente "En la distribución B". De $21.6 \%$ que acertó, $9.01 \%$ correspondió a costarricenses y el restante $12.61 \%$ a mexicanos. La Figura 2 muestra los resultados obtenidos y un comparativo; presenta los resultados agrupados por nación, la cantidad de respuestas correctas y su proporción respecto al total. El bajo desempeño obtenido y el tipo de errores cometidos indican una posible dificultad para leer e interpretar con precisión información en formato discontinuo, entendiendo este término como la habilidad de un individuo para descifrar y explicar gráficos, esquemas y tablas, entre otras representaciones intrínsecas a técnicas visuales de uso común en estadística. 


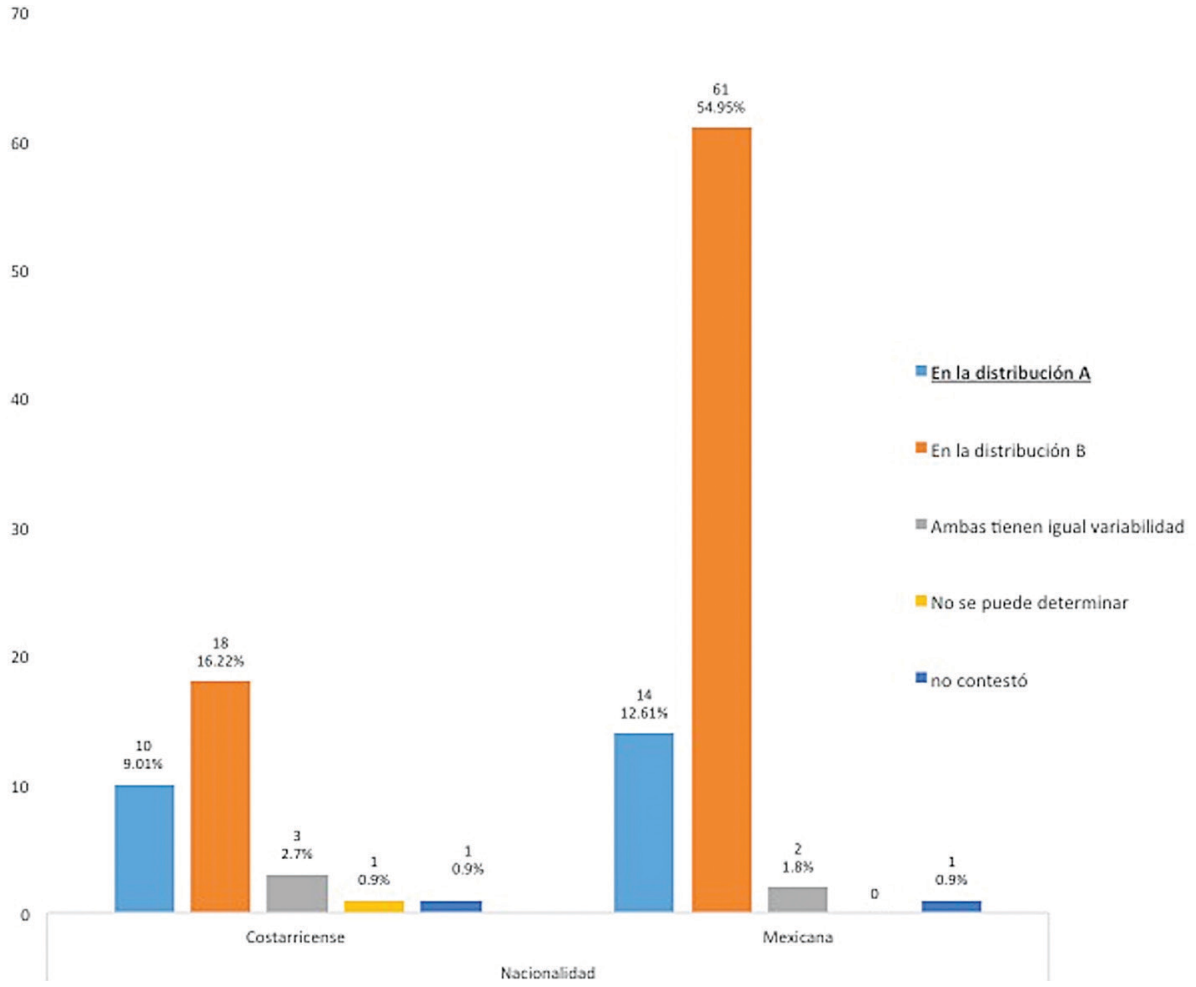

Figura 2. Respuestas del ítem sobre variabilidad de distribuciones.

En los ítems 4 y 5 (Figuras 3 y 4, respectivamente) se evaluó el desempeño en lectura e interpretación de gráficas de caja. En el ítem 4 (nivel 3 de PISA), 43.2\% respondió correctamente. La proporción de aciertos por región fue de 39.3\% y 44.8\% para costarricenses y mexicanos, respectivamente.

En el ítem 5 (nivel 3, PISA) se presentaron resultados similares. Únicamente 41.4\% de los 111 participantes afirmaron correctamente que "La distribución de las estaturas presenta un sesgo a la izquierda". Durante el proceso de aplicación de la prueba varios profesores indicaron que no habían utilizado con antelación este tipo de representación gráfica, aunque señalaron que la habían observado en algunos textos. 
40

35

25

20

15

(n)

30

10

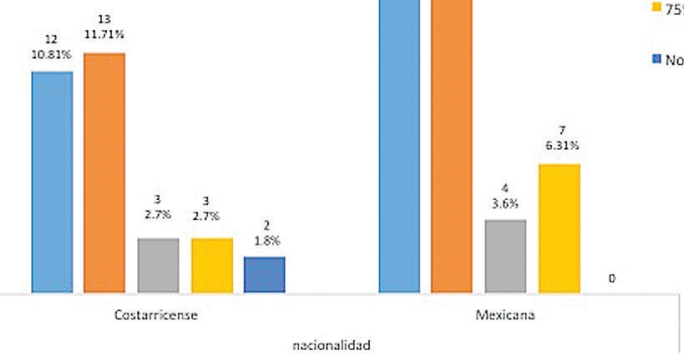

\# El promedio de las estaturas es de $163 \mathrm{~cm}$

$=75 \%$ de las estaturas es de $163 \mathrm{~cm}$ a menes

$=50 \%$ de las estaturas es de $163 \mathrm{~cm}$

$75 \%$ de las estaturas es de $163 \mathrm{~cm}$ o más

No contestó

nasionalidad

Figura 3. Respuestas del ítem sobre interpretación de diagrama de caja.

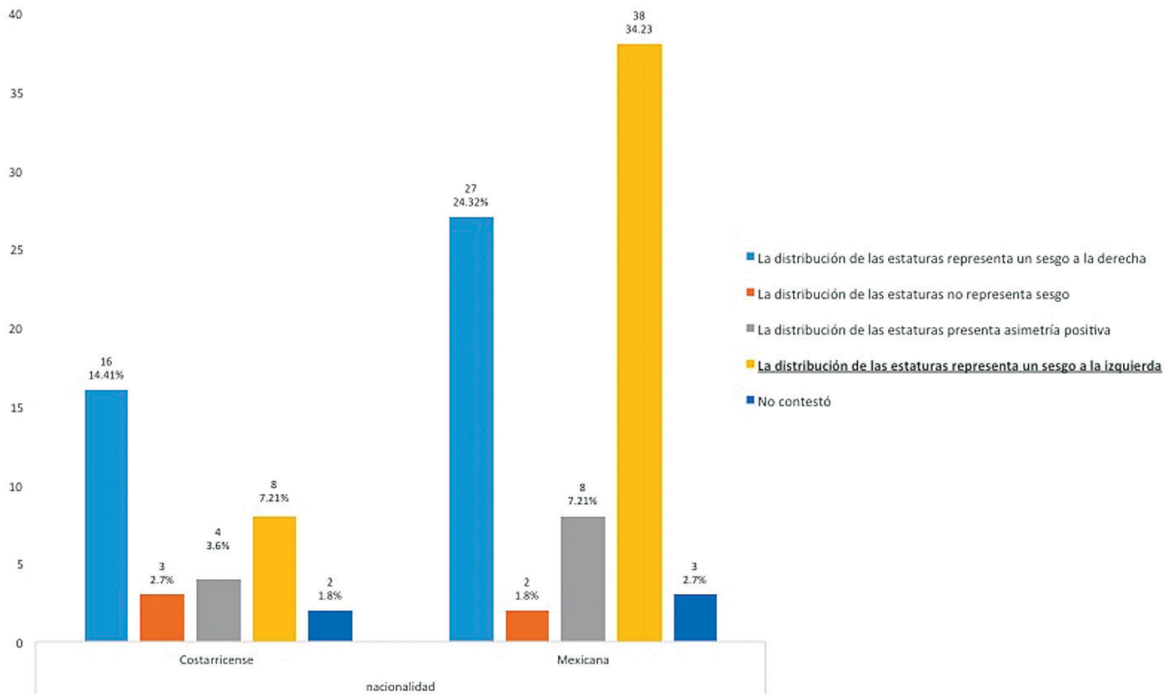

Figura 4. Respuestas del ítem sobre sesgo de una distribución. 
Para leer e interpretar datos agrupados en tablas se diseñó el ítem 7 (nivel 4 de PISA). Los resultados obtenidos señalan que únicamente $14.4 \%$ de los participantes contestó correctamente lo solicitado, según la Figura 5.

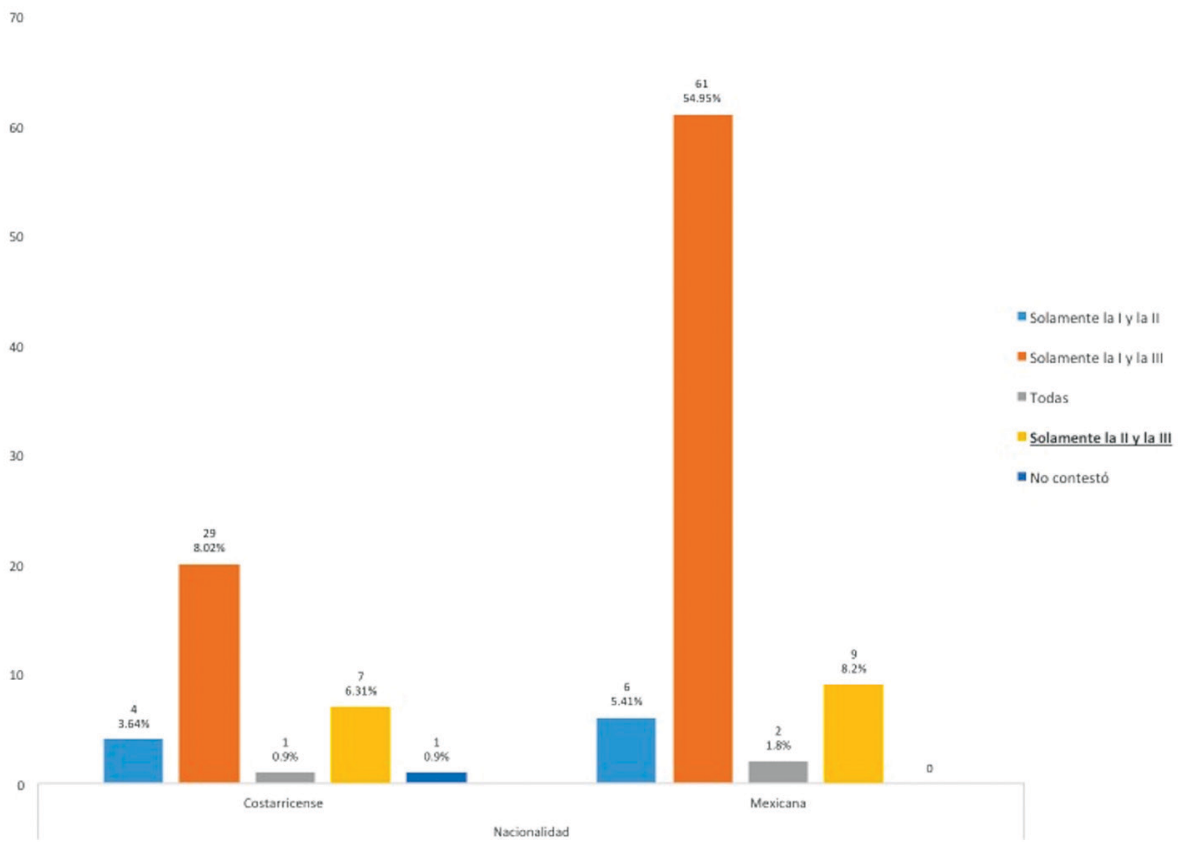

Figura 5. Respuestas del ítem sobre la interpretación de medidas de tendencia central.

El ítem 9 (nivel 4 de acuerdo con PISA) exigió efectuar un análisis gráfico para calcular probabilidades con porcentajes. Se encontró, según muestra la Figura 6, que $72.98 \%$ de los docentes seleccionó la respuesta correcta, $84.8 \%$ y $67.94 \%$ para costarricenses y mexicanos, respectivamente.

No existe evidencia significativa para rechazar que las proporciones de aciertos en esta temática sean iguales entre ambas naciones. 


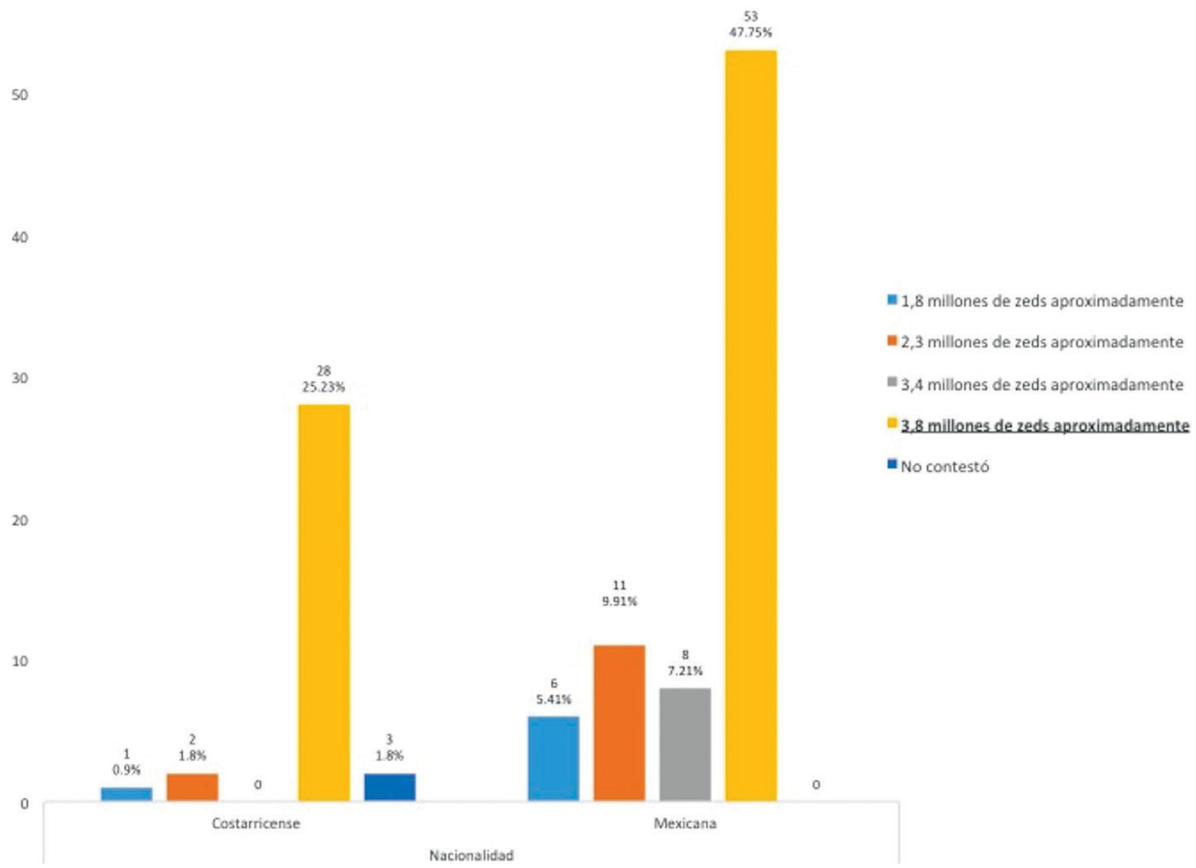

Figura 6. Respuestas del ítem sobre la interpretación de un gráfico de barras y circular.

\subsection{EXPERIMENTOS ALEATORIOS Y DETERMINISTAS}

Respecto al ítem 2 sobre la probabilidad de ocurrencia de un evento, 94.6\% de los profesores respondieron correctamente; por nación, 96.9\% de los costarricenses y $93.58 \%$ de los mexicanos acertó. Este ítem alcanzó el nivel 1 en la escala PISA, y fue el único de su tipo en el instrumento para medir el desempeño disciplinar. Se esperaba que todos los participantes acertaran en su respuesta, sin embargo, cuatro de ellos la equivocaron y dos no contestaron, según detalla en la siguiente figura. 


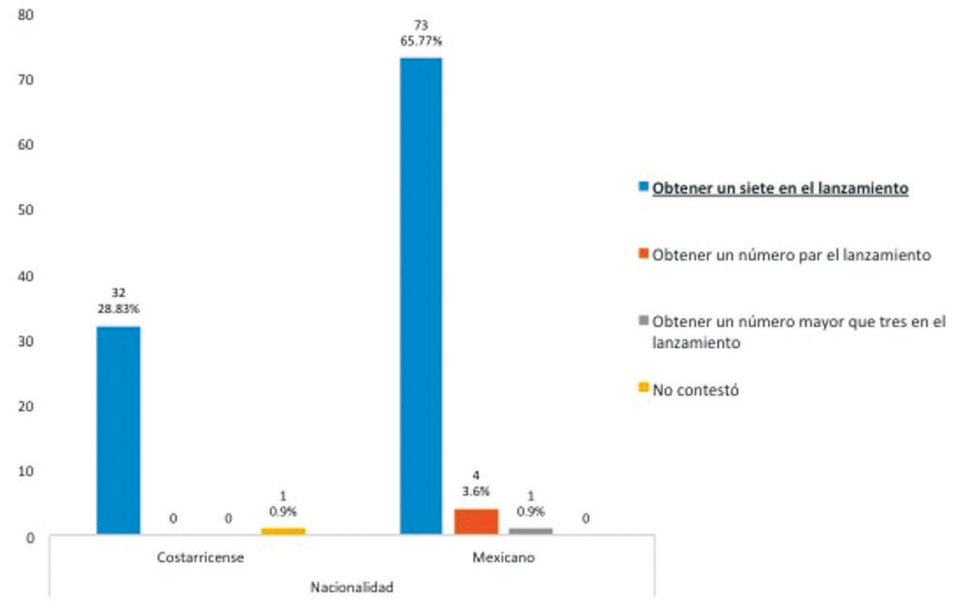

Figura 7. Respuestas del ítem sobre lo que es un evento imposible.

Con el ítem 3 se indagó en qué medida los docentes podían identificar las diferencias entre fenómenos aleatorios y deterministas. De acuerdo con los parámetros de la prueba PISA, este ítem pertenece al nivel 3 en términos de dominio exigido. 71.2\% de todos los profesores seleccionó la respuesta correcta, 57.5\% y 76.9\% para costarricenses y mexicanos, respectivamente, como muestra la Figura 8.

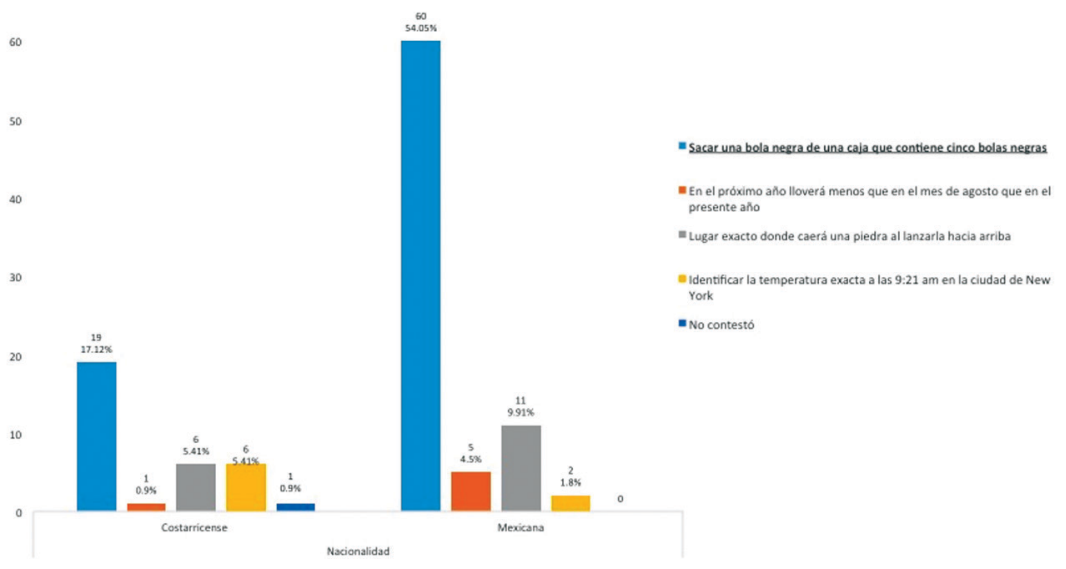

Figura 8. Respuestas del ítem sobre lo que es un evento determinista. 
Aunque hay una leve ventaja de los profesores de Chihuahua en la proporción de aciertos de esta temática, no es significativa.

\subsection{MEDIDAS DE TENDENCIA CENTRAL}

El ítem 6 (nivel 2 de PISA) planteó una situación que demandó interpretar cuál medida de tendencia central debía usarse para responder una interrogante; fue resuelto satisfactoriamente por $48.6 \%$ de los participantes, $45.4 \%$ y $50 \%$ para costarricenses y mexicanos, respectivamente (ver Figura 9).

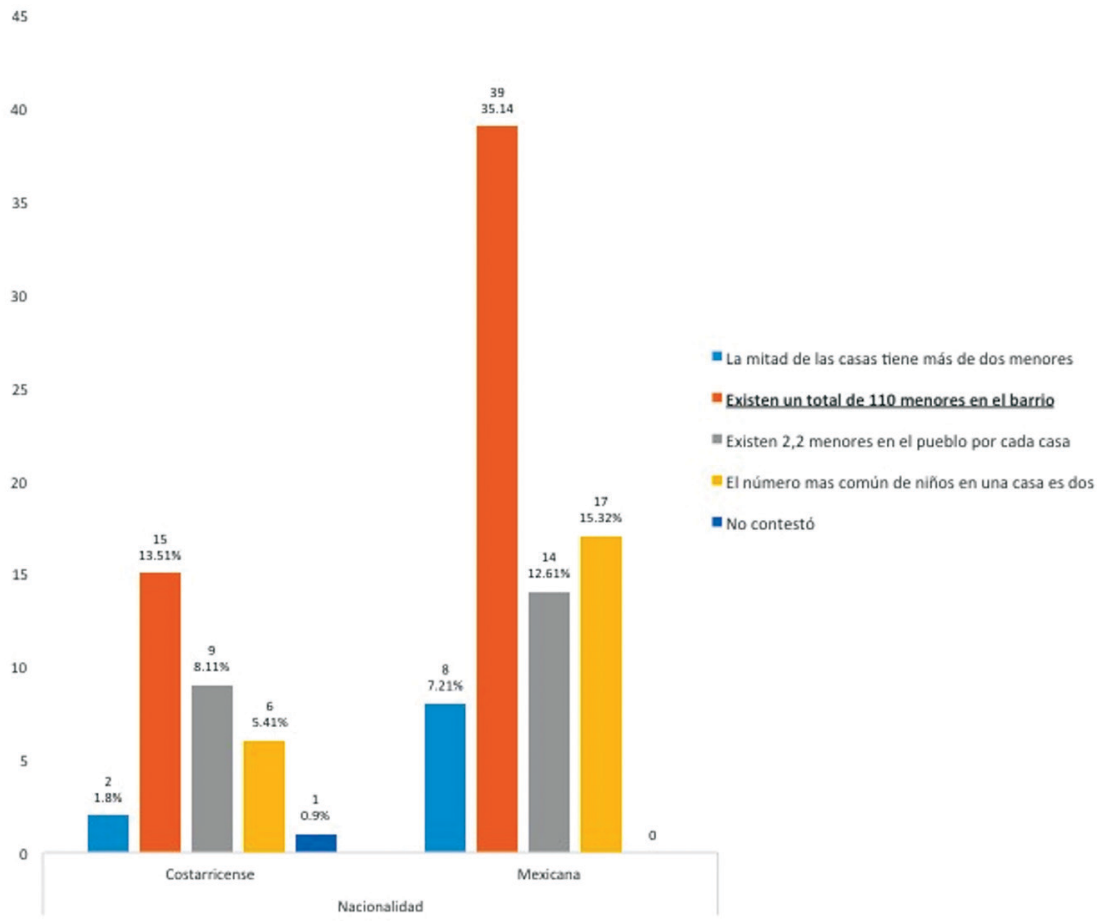

Figura 9. Respuestas del ítem sobre el significado del promedio.

El ítem 8 fue uno de los que mayor nivel (5) alcanzó en la escala PISA. En él se solicitó examinar una situación que mostraba una serie de afirmaciones que 
evaluaban la interpretación de medidas de tendencia central. Únicamente 39.64\% seleccionó la respuesta correcta. La Figura 10 muestra algunos resultados representativos.

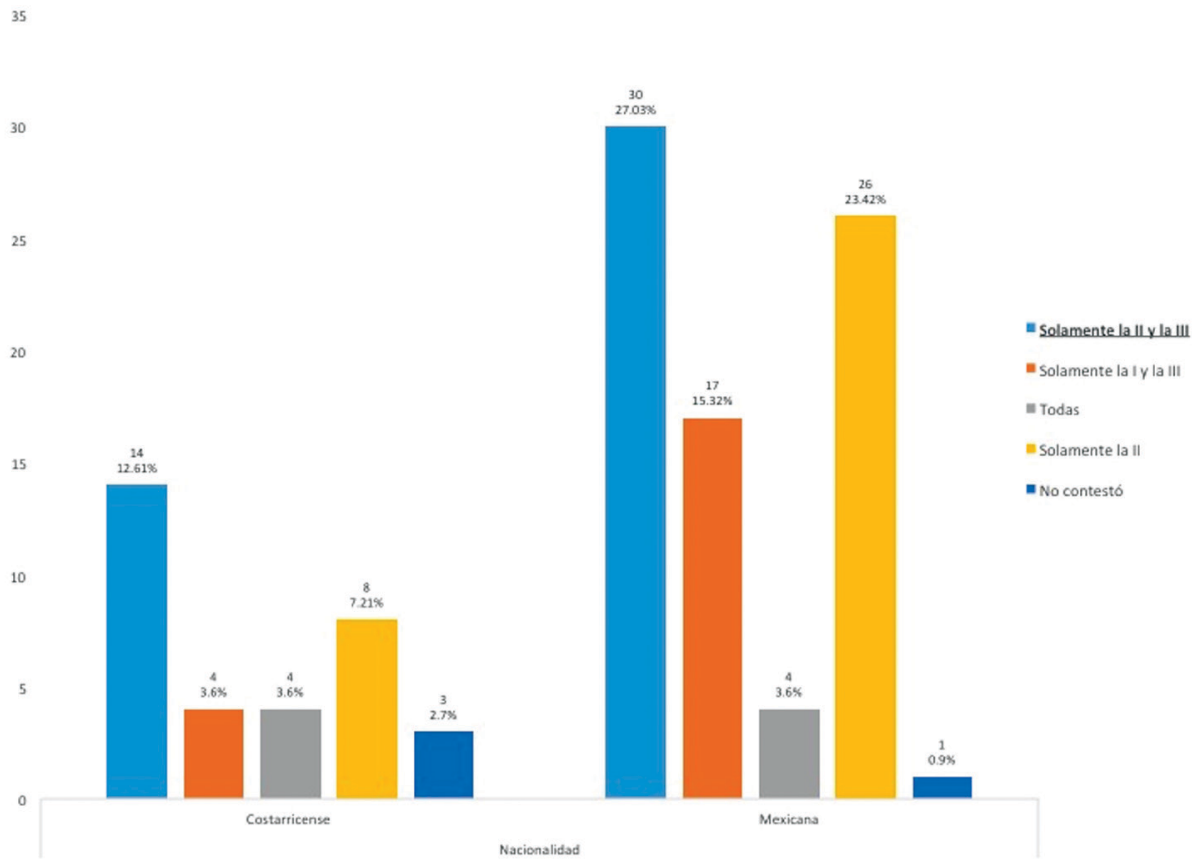

Figura 10. Respuestas del ítem sobre medidas de tendencia central y variabilidad.

El ítem 11 presentó una situación que exigió observar dos figuras y seleccionar la medida de posición central que mejor resumiera la ubicación de la concentración mayor de datos. El ítem tuvo un nivel de dificultad 2 de acuerdo a los parámetros de PISA y aun así, solo 44.14\% respondió con acierto. 


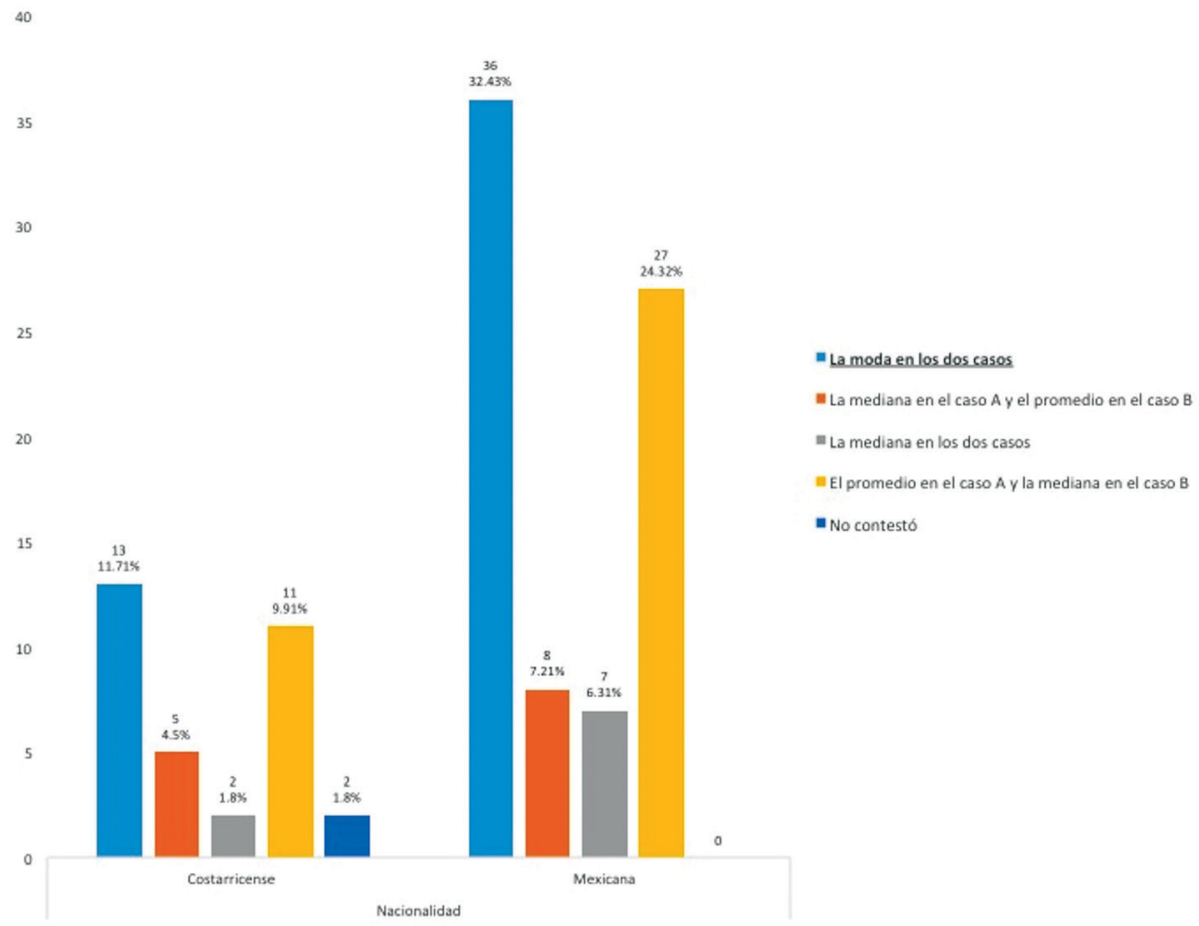

Figura 11. Respuestas del ítem sobre una distribución bimodal.

Las proporciones de aciertos en esta temática son similares para ambas naciones.

\subsection{CLASIFICACIÓN DE VARIABLES}

Los ítems 12, 13 y 14 (nivel 3, PISA) fueron configurados para examinar en qué medida los profesores clasificaban variables y seleccionaban la medida de tendencia central y la representación gráfica adecuadas. Los resultados en el ítem 12 (Figura 12) indican que solo 36.9\% de los participantes acertaron en su respuesta. Erróneamente, para la variable "sexo" cerca de 40\% indicó que era de tipo cuantitativa, que se le podría calcular la media aritmética y que la gráfica de barras era la más adecuada para representar los datos. 


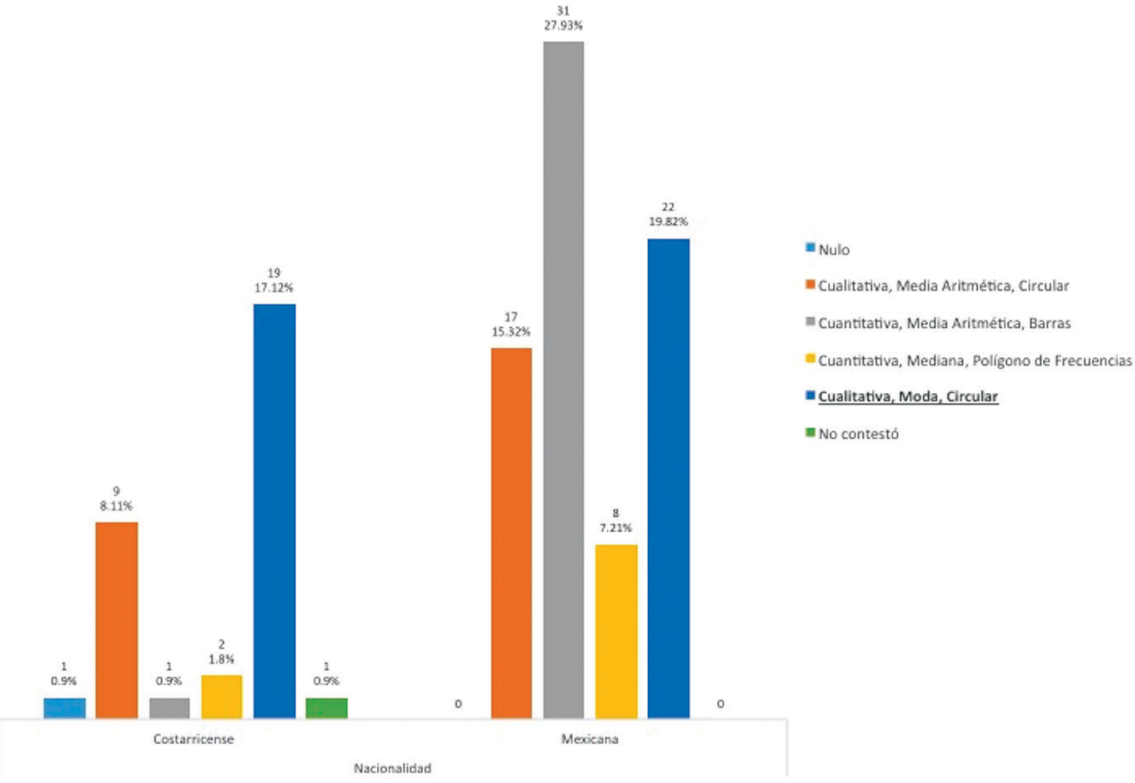

Figura 12. Respuestas del ítem sobre la clasificación de la variable "sexo".

El ítem 13 (Figura 13) solicitó clasificar la variable "número de habitaciones". $45.9 \%$ de los docentes acertó en su respuesta. Por región, 54.5\% de los costarricenses y $42.30 \%$ de mexicanos estuvieron en la misma situación. 


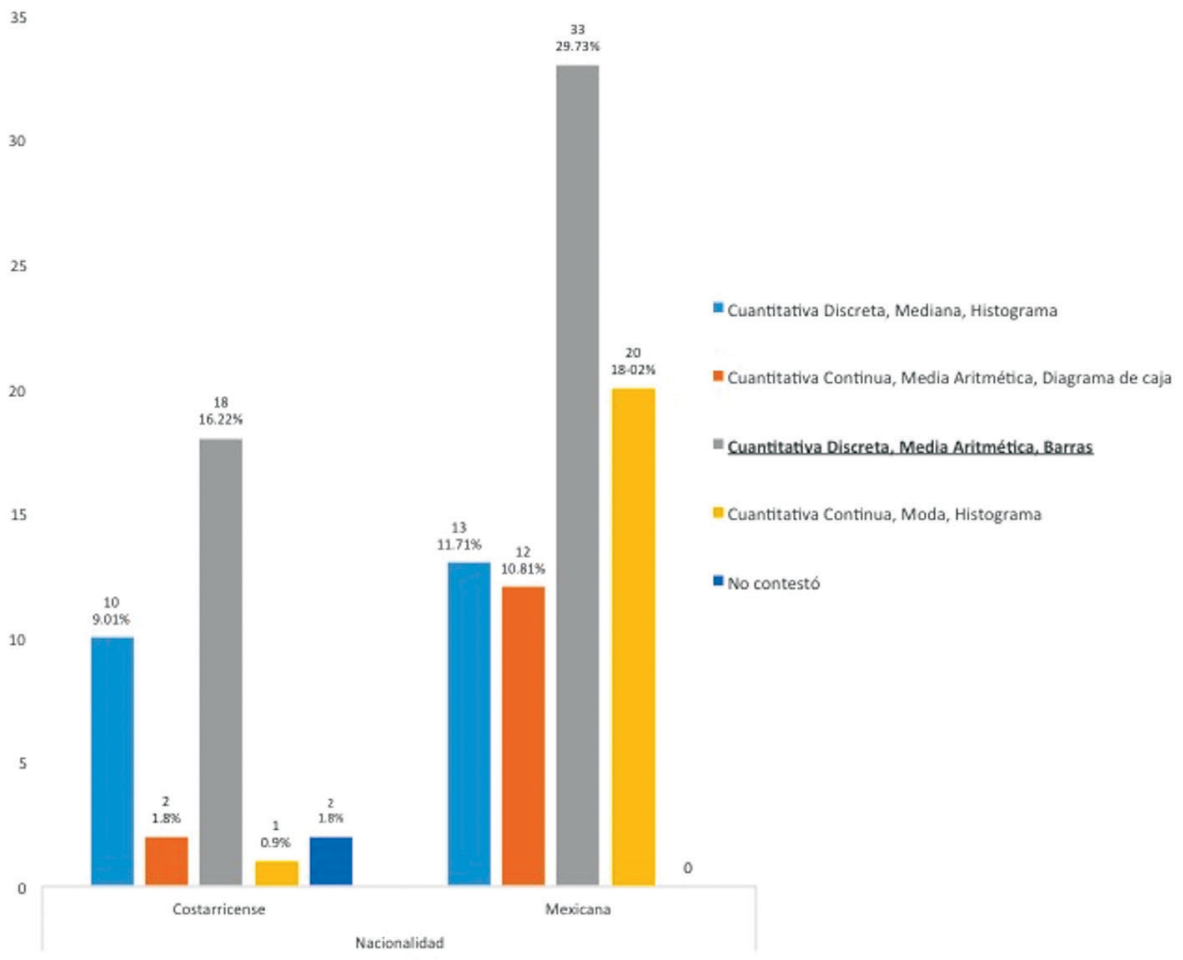

Figura 13. Respuestas del ítem sobre la clasificación de la variable "número de habitaciones".

El desempeño en el ítem 14 (Figura 14) siguió el mismo patrón que los ítems anteriores, solo 34.2\% lo resolvió correctamente. En esa misma condición quedaron por nación $60.6 \%$ y $38.46 \%$ de costarricenses y mexicanos, respectivamente. 


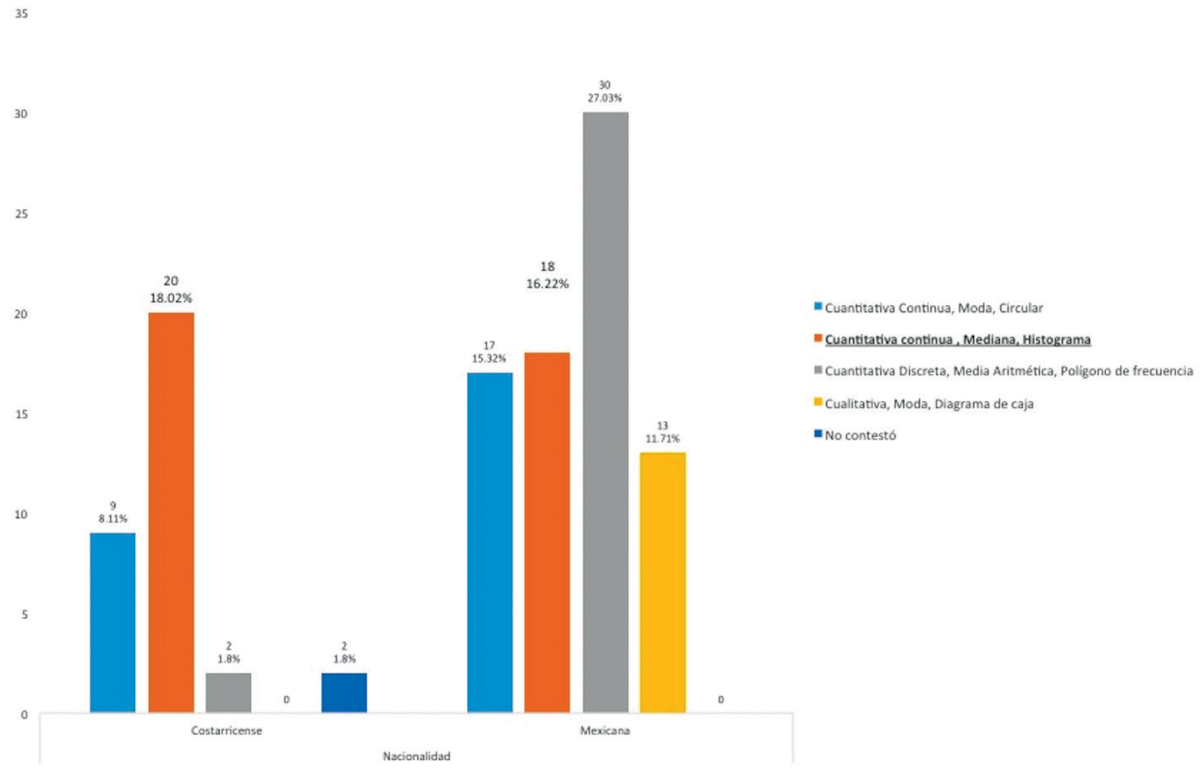

Figura 14. Respuestas del ítem sobre la clasificación de la variable "peso".

Hay evidencia significativa (0.081) para rechazar la hipótesis de que las proporciones de acierto en esta temática son iguales para los grupos de profesores de ambas nacionalidades, los costarricenses superan a los mexicanos.

\subsection{Probabilidad}

Algunos programas de estudio establecen introducir a los alumnos en el aprendizaje de la probabilidad condicional y, en consecuencia, fue confeccionado un ítem que evalúa el desempeño en este tema. El ítem 6 alcanzó el nivel máximo de dificultad en la escala PISA. Solamente $46.85 \%$ contestó con acierto y por nación lo hicieron $54.5 \%$ de los costarricenses y $43.58 \%$ de los mexicanos, de acuerdo con la siguiente figura. 


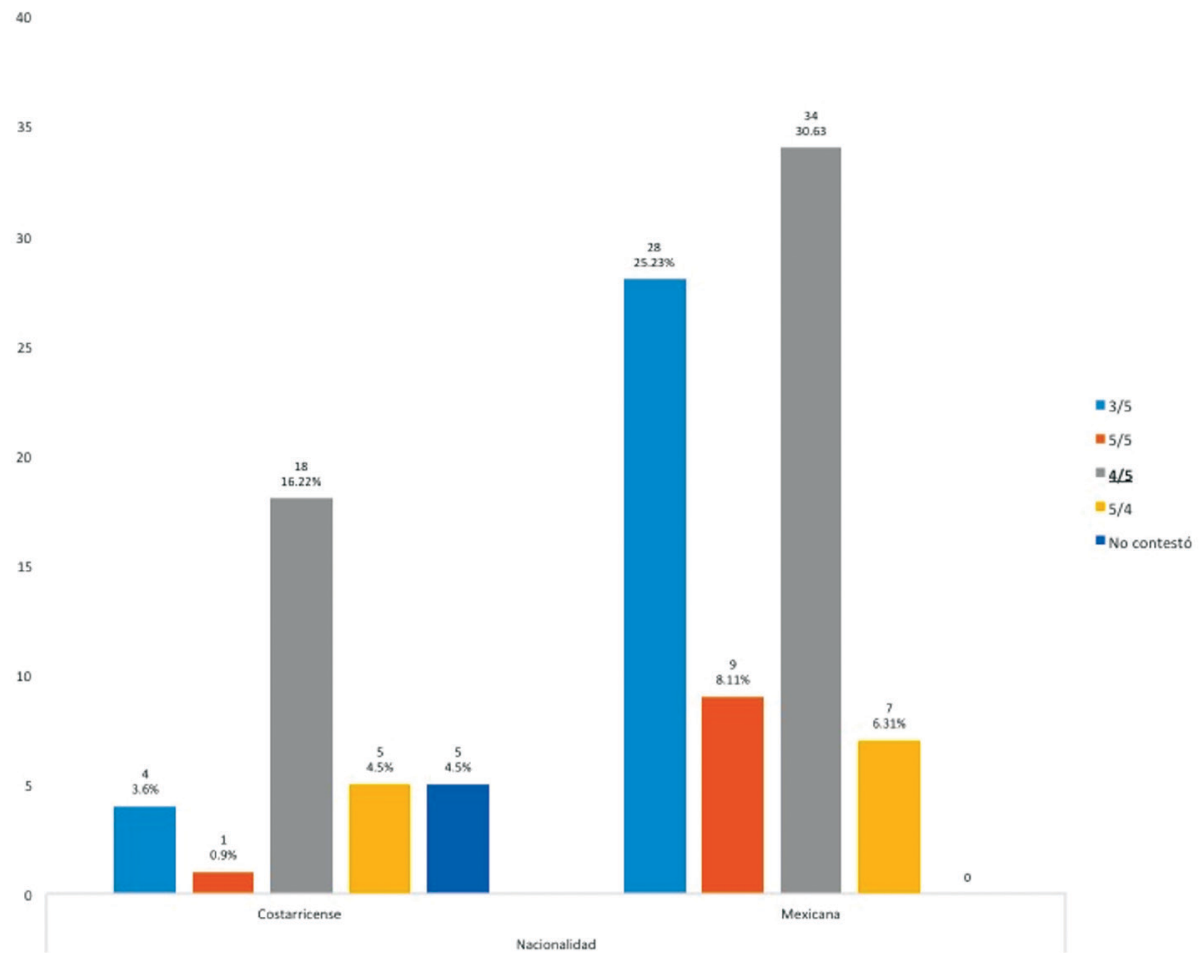

Figura 15. Respuestas del ítem sobre la probabilidad condicional.

\subsection{DiagRama DE GolBaCK E INDEPENDENCIA DE EVENTOS}

Los ítems 15 (nivel 5 según PISA) y 16 (nivel 4 de PISA) exigieron resolverlos, redactar el procedimiento seguido y justificar la respuesta. En el ítem 15 se presentó un Diagrama de Golback que simuló lanzar 100 bolas que caerían sobre depósitos, se hizo una pregunta sobre el cálculo de probabilidades y los profesores debían justificar la respuesta. En el ítem 16 se planteó una situación relacionada con el tema de eventos independientes.

En el ítem 15 (Figura 16), los resultados fueron mixtos: 74.77\% de los participantes respondió correctamente la pregunta, pero no todos justificaron su respuesta con acierto. 
Los resultados obtenidos en el ítem 16 (Figura 17) señalan que 79.28\%seleccionó la opción correcta, $13.51 \%$ la incorrecta y el restante $7.21 \%$ no contestó. El análisis por nación indica que $63.6 \%$ de los costarricenses y $85.89 \%$ de los mexicanos acertó, pero $21.2 \%$ de los costarricenses no emitió respuesta alguna.

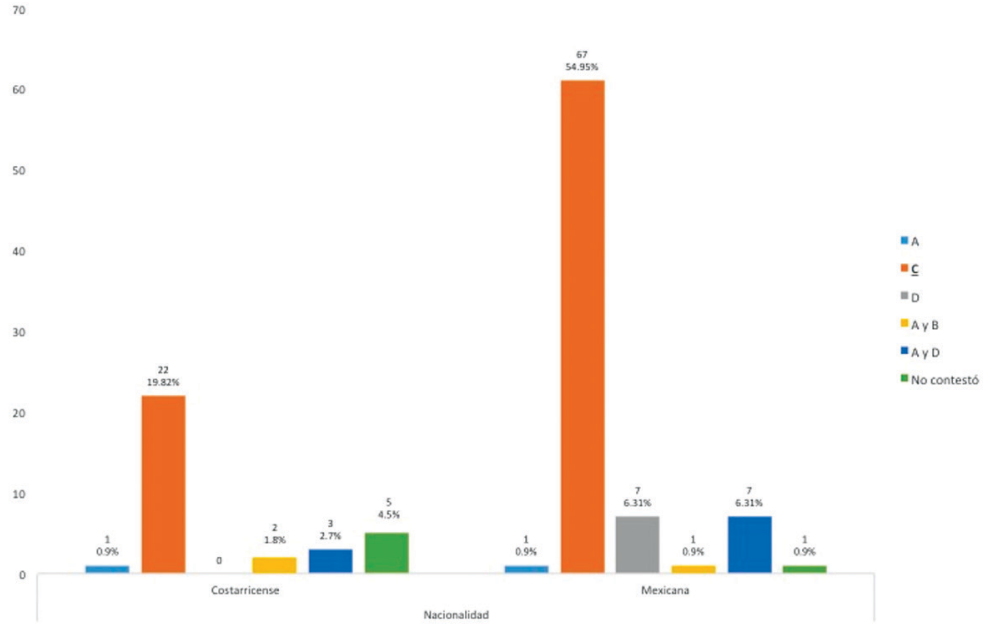

Figura 16. Respuestas del item sobre el diagrama de Golback.

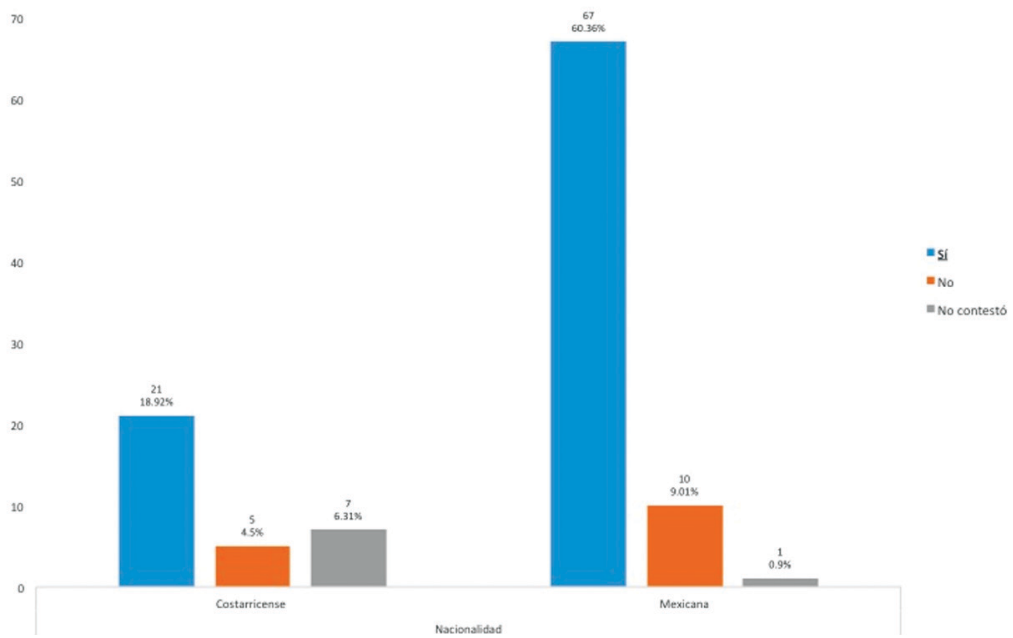

Figura 17. Respuestas del ítem sobre independencia de eventos. 
No hay diferencias significativas entre las proporciones de acierto para los profesores de ambas naciones en el ítem 6 de probabilidad y el ítem 15 sobre el diagrama de Golback.

Entre tanto, la proporción de acierto en el ítem 16 es superior en los chihuahuenses que en los cartagineses de manera significativa (valor $p=0.0082$ ).

Respecto a las interrogantes planteadas líneas atrás, a saber, ¿cuál es el grado de dominio en estocástica?, ¿qué características comparten en términos de dominio? y ċen qué medida dicho dominio es suficiente para cumplir con los estándares curriculares exigidos en los programas de estudio?, las respuestas pueden enunciarse de la siguiente manera: el desempeño de los profesores participantes de ambas naciones fue bajo. El nivel de dominio mostrado no es suficiente para aspirar a cumplir con los estándares educativos en estocástica establecidos en los programas de estudio entre el séptimo y duodécimo grado; una proporción alta de docentes desconoce los principios básicos de la probabilidad, no clasifican variables de manera adecuada y les resulta difícil leer e interpretar los datos agrupados en tablas y gráficas. Fue sorprendente encontrar que una proporción significativa de participantes tiene problemas en diferenciar entre fenómenos aleatorios y deterministas (19 profesores), un tópico irreductible para cualquier docente -o aspirante a serlo-, especialmente en los niveles educativos iniciales, donde se debe comenzar a formar a los estudiantes en estos temas. Hubo pequeñas diferencias en el desempeño entre costarricenses y mexicanos, principalmente en los tópicos de clasificación de variables, donde los cartagineses tuvieron mejor rendimiento, entre tanto los chihuahuenses aventajaron en el ítem correspondiente a la independencia de eventos.

\section{CONCLUSIONES Y RECOMENDACIONES}

Sin considerar su ubicación geográfica, número de habitantes, estructura del sistema educativo y variables de otra índole, Costa Rica y México son naciones que han adoptado estándares educativos internacionales que se reflejan en sus currículos escolares y en el énfasis por implantar programas de formación académica para su profesorado.

Clasificar variables, analizar, presentar y tratar información estadística en formato continuo y discontinuo, identificar diferencias entre fenómenos aleatorios y deterministas, seleccionar medidas de tendencia central, efectuar cálculos 
básicos de probabilidad e interpretar diagramas constituyen habilidades y saberes irreductibles que deben reunir los docentes.

El estudio exploró y comparó el desempeño de profesores de ambas naciones en relación a dichos tópicos. Los resultados obtenidos indican un bajo desempeño en la interpretación de las tablas y gráficos estadísticos, la clasificación de variables y la selección apropiada de medidas de tendencia central. Por otra parte, los profesores tuvieron un desempeño adecuado en la identificación de fenómenos aleatorios de los que no lo son.

Los resultados presentados no son concluyentes. Como se indicó, el estudio fue exploratorio; sin embargo, las respuestas de los 111 participantes dan un indicio de cuál es la situación actual y permitirán derivar hipótesis susceptibles de ser probadas empíricamente en estudios más amplios.

En respuesta a la pregunta ¿cuáles son las principales áreas de mejora o necesidades de actualización que presenta el profesorado? se recomienda que las instituciones diseñen programas de trabajo para que los docentes sean capaces de: 1) Conocer el desarrollo histórico de la estadística y la probabilidad, 2) Entender los postulados probabilísticos más representativos, 3) Comprender y usar el lenguaje propio de estas disciplinas, 4) Leer e interpretar información presentada en tablas y gráficas, 5) Seleccionar y utilizar de forma coherente diferentes medios para representar datos, 6) Resolver problemas de tipo estocástico dentro y fuera del ámbito escolar, 7) Emitir conclusiones y recomendaciones que respeten la lógica de trabajo y el alcance del análisis estadístico.

Es necesario que las instituciones examinen la vigencia y prospectiva de sus programas de formación, actualización y capacitación de profesores. Este examen puede guiarse a través del planteamiento de interrogantes como las siguientes: ¿en qué medida el perfil de egreso actual es acorde con las necesidades y fines académicos en el plano nacional e internacional?, ¿qué características debe reunir el perfil de egreso a plasmar en los planes de estudio? y ¿cómo pueden articularse programas de formación, actualización y capacitación que respondan a las demandas sociales y científicas en las que se privilegie el logro de aprendizajes de calidad?

Nota: Una versión reducida de este documento se publicó en el $13^{\text {th }}$ International Congress on Mathematical Education (ICME 13) celebrado en Hamburgo Alemania, 2016. http://iase-web.org/documents/papers/icme13/ICME13_S2_Cuevas.pdf. 


\section{AGRADECIMIENTO SOBRE LA FINANCIACIÓN}

La realización de esta investigación contó con el auspicio del Departamento de Ciencias Básicas del Instituto Tecnológico de Chihuahua ॥ (México) y la Escuela de Matemática del Instituto Tecnológico de Costa Rica.

\section{REFERENCIAS}

Alsina, A. y Vásquez, C. (2016). Análisis de los conocimientos probabilísticos del profesorado de Educación Primaria. Revista Digital: Matemática, Educación e Internet, 16(1). Consultado el 12 de enero de 2016 en: http://doi.org/10.18845/rdmei.v16i1.2475-

Ballestero, E. (2006). Pensamiento estadístico que muestran los profesores al estudiar la centralidad y la variabilidad en un contexto de estadística dinámica con tecnología. México: Centro de Investigación y Estudios Avanzados, IPN, 2006. 245 p.

Barquín, J., Gallardo, M., Fernández, M., Yus, R., Sepúlveda, M. y Serván, M. (2011). Todos queremos ser Finlandia: los efectos secundarios de PISA. Education in the Knowledge Society (EKS), 12(1), pp. 320-339. Consultado el 29 de enero de 2016 en: http://campus.usal.es/ revistas_trabajo/index.php/revistatesi/article/view/7835/7861.

Batanero, C., Arteaga, P. y Contreras, J. (2011). El currículo de estadística en la enseñanza obligatoria. Revista de Educação Matemática e Tecnológica Iberoamericana, 2(2). Consultado el 3 de enero de 2016 en: http://www.ugr.es/ jmcontreras/pages/Investigacion/articulos/2011EmTEia.pdf.

Biehler, R. (2016). Professional Development for Teaching Probability and Inference Statistics with Digital Tools at Upper Secondary Level. $13^{\text {th }}$ International Congress on Mathematical Education ICME 13, Hamburgo, Alemania.

Estrella, S., Olfos, R. y Mena-Lorca, A. (2015). El conocimiento pedagógico del contenido de estadística en profesores de primaria. Educação e Pesquisa, 41(2), 477-493. http:// doi.org/10.1590/S1517-97022015041858.

Garfield, J. delMas, R. y Chance, B. (2004). Reasoning about Sampling Distributions. En: D. Ben-Zvi y J. Garfield (eds.), The Challenge of Developing Statistical Literacy, Reasoning and Thinking (pp. 295-323). Netherlands: Kluwer Academic Publishers.

Huerta, P. (2016). Preparing Teachers for Teaching Probability through Problem Solving. $13^{\text {th }}$ International Congress on Mathematical Education ICME 13. Hamburgo, Alemania.

INEE (2005). PISA para docentes: La evaluación como oportunidad de aprendizaje. México: Instituto Nacional para la Evaluación de la Educación. 
Insunza, S. y Guzmán, M. (2011). Comprensión que muestran profesores de secundaria acerca de los conceptos de probabilidad: un estudio exploratorio. Educación Matemática, 23(1), pp. 63-95. Consultado el 1 de febrero de 2016 en: http://www.redalyc. org/pdf/405/40521127003.pdf.

Marz, V. y Kelchtermans, G. (2013). Sense Making and Structure in Teachers Reception of Educational Reform. A Case Study on Statistics in the Mathematics Curriculum. Teaching and Teacher Education, 29, 13-24. DOl: http://dx.doi.org/10.1016/j. tate.2012.08.004.

Meneses, S. (1993). Análisis de ítems de opción múltiple en matemática. Las matemáticas y su enseñanza, 5(14).

MEP (2012). Reforma curricular en Ética, Estética y Ciudadanía. Programas de Estudio de Matemática I y II ciclo de Educación Primaria, III ciclo de Educación General Básica y Diversificada. San José, Costa Rica: Ministerio de Educación Pública.

OCDE (2004). Learning for Tomorrow's World First Results from PISA 2003. París, Francia: Organización para la Cooperación y el Desarrollo Económico.

Pedró, F. (2012). Deconstruyendo los puentes de PISA: del análisis de resultados a la prescripción política. Revista Española de Educación Comparada, 0(19), 139-174. Consultado el 19 de septiembre de 2015 en: http://dx.doi.org/10.5944/reec.19.2012.7581. SEP (2011). Plan de estudios 2011. Educación Básica. México. Secretaría de Educación Pública.

Shaughnessy, J. (2007). Research on Statistics Learning, En: Second Handbook of Research on Mathematics Teaching and Learning (pp. 957-1009). EUA: NCTM.

Watson, J. (2013). Statistical Literacy at School: Growth and Goals. EUA: Routledge.

Zieffler, A., Park, J., Garfield, J., Delmas, R. y Bjornsdottir, A. (2012). The Statistics Teaching Inventory: A Survey on Statistics Teachers Classroom Practices and Beliefs. Journal of Statistics Education, 1(12), pp. 1-29. Consultado el 14 de noviembre de 2015 en: https://ww2.amstat.org/publications/jse/v20n1/zieffler.pdf. 


\section{Anexo 1. Instrumento}

\section{Cuestionario dimensión disciplinar}

\subsection{INSTRUCCIONES GENERALES}

El cuestionario que usted tiene en sus manos tiene el propósito de obtener información sobre su grado de dominio en solución de problemas en las áreas de probabilidad y estadística. Solicitamos atentamente su ayuda dando respuesta a lo que se le pide. No es necesario que anote su nombre. La información recabada será tratada de forma estrictamente confidencial y servirá para generar propuestas de apoyo que incidan en la adecuación y creación de programas de capacitación, actualización de profesores en activo y en proceso de formación.

Parte I: Selección Única. Marque con una equis (X) la opción que corresponde a la respuesta correcta. Debe marcar solo una opción por pregunta.

1. ¿Cuál de las siguientes distribuciones tiene más variabilidad?
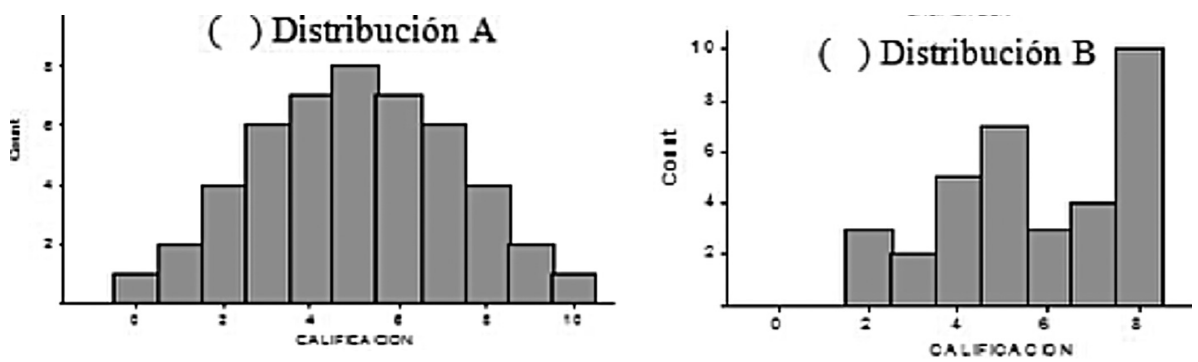
( ) En la distribución A
( ) En la distribución B
( ) Ambas tienen igual variabilidad
( ) No se puede determinar 
2. Un estudiante lanza un dado justo de seis caras numeradas del 1 al 6 , el siguiente evento es imposible:
( ) Obtener un siete en el lanzamiento
( ) Obtener un número par en el lanzamiento
( ) Ambas tienen igual variabilidad
( ) No se puede determinar

3. Se desea realizar un análisis sobre las diferencias entre experimentos aleatorios y determinista (no aleatorios). ¿Cuál de los siguientes experimentos es un ejemplo de una situación determinista?
( ) Sacar una bola negra de una caja que contiene cinco bolas negras
( ) En el próximo año lloverá menos en el mes de agosto que en el presente año
( ) Lugar exacto donde caerá una piedra al lanzarla hacia arriba
( ) Identificar la temperatura exacta a las 9:21 am en la ciudad de New York

4. Un estudio referente a estaturas en jóvenes arrojó la siguiente representación gráfica.

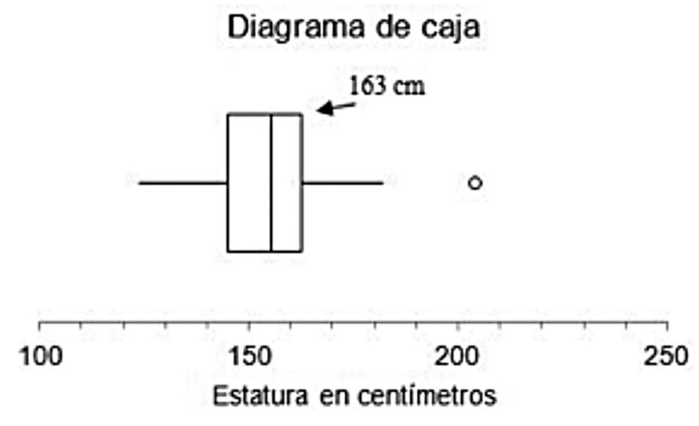

( ) El promedio de las estaturas es de $163 \mathrm{~cm}$

( ) $75 \%$ de las estaturas es de $163 \mathrm{~cm}$ o menos

( ) $50 \%$ de las estaturas es de $163 \mathrm{~cm}$

( ) $75 \%$ de las estaturas es de $163 \mathrm{~cm}$ o más 
5. Con base en la gráfica presentada en el ítem anterior (4), se puede afirmar que:

( ) La distribución de las esturas presenta un sesgo a la derecha

( ) La distribución de las estaturas no presenta sesgo

(.) La distribución de las estaturas presenta asimetría positiva

\section{( ) La distribución de las estaturas presenta un sesgo a la izquierda}

6. En un pequeño barrio hay un total de 50 casas. El comité de bienestar estudiantil de este barrio ha decidido determinar el número promedio de menores que hay por casa en este barrio. Los encargados del comité dividieron el total de infantes entre 50 y el promedio de menores que obtuvieron fue de 2,2. ¿Cuál de las siguientes afirmaciones considera que es siempre verdadera?

( ) La mitad de las casas tiene más de dos menores

( ) Existe un total de 110 menores en el barrio

( ) Existen 2,2 menores en el pueblo por cada casa

( ) El número más común de niños en una casa es dos

7. En el cuadro siguiente se muestran los años de experiencia que tienen 196 docentes de educación primaria que fueron encuestados en el 2009

\begin{tabular}{|c|c|c|}
\hline Años de experiencia & Núm.docentes & \% docentes \\
\hline De 1 a menos 5 & 28 & 14,285 \\
\hline De 5 a menos 10 & 31 & 15,816 \\
\hline De 10 a menos de 15 & 37 & 18,875 \\
\hline De 15 a menos 20 & 40 & 20,408 \\
\hline De 20 a menos 25 & 48 & 24,489 \\
\hline De 25 a menos 30 & 12 & 6,122 \\
\hline Total & 196 & 100 \\
\hline
\end{tabular}

Con base en los datos del cuadro anterior, analice las siguientes proposiciones: 
I. La mayoría de los docentes de primaria encuestados tiene más de 20 y menos de 25 años de experiencia en labores docentes.

II. Aproximadamente 39.3\% de los docentes tienen 10 años o más, pero menos de 20 de laborar en educación primaria.

III. El valor de la mediana en relación a la experiencia de los docentes se encuentra entre 15 y menos de 20 años.

De las proposiciones anteriores, son verdaderas:

( ) Solamente la I y la II

( ) Solamente la I y la III

( ) Todas

( ) Solamente la II y la III

8. El ingreso promedio mensual de los empleados de una institución que tiene 1350 empleados es \$1 600 y la mediana de los ingresos es \$1 000: Analice las siguientes afirmaciones:

I. El monto total que paga la institución en salarios al mes es aproximadamente \$1350000

II. La mediana es más representativa de la distribución de los salarios que el promedio.

III. En esta institución hay empleados que tienen salarios muy altos con respecto al total de los trabajadores.

\section{( ) Solamente la II y la III}

( ) Solamente la I y la III

( ) Todas

( ) Solamente la II

9. En relación al siguiente gráfico, ¿̇uál es el valor del jugo de fruta exportado de Zedlandia en el año 2000? 
Total annual exports from Zedland in millions of zeds, 1996-2000

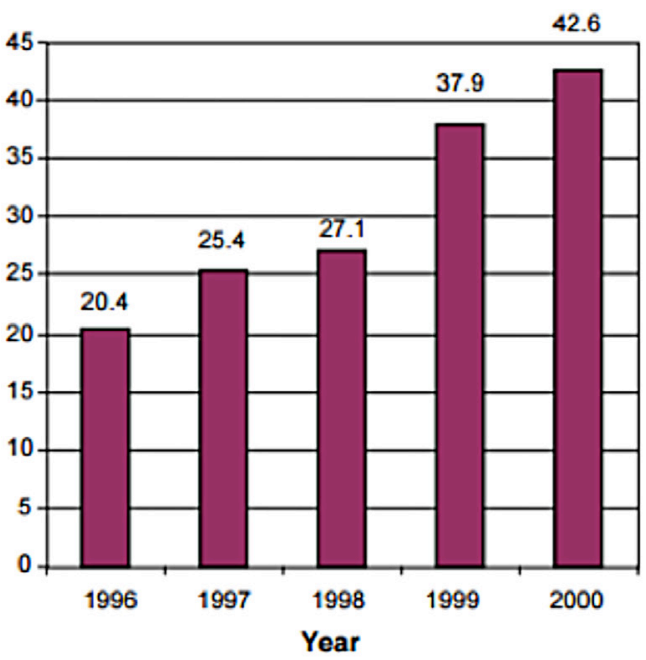

Distribution of exports from Zedland in $\mathbf{2 0 0 0}$

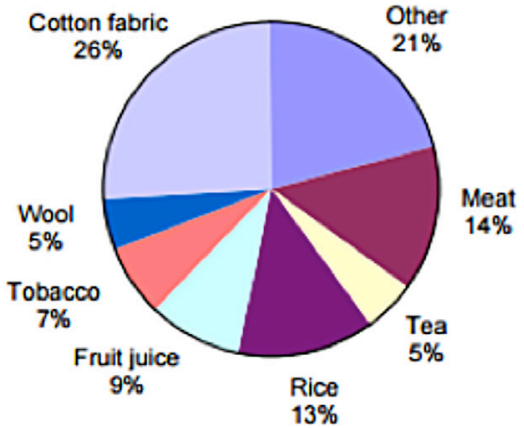

( ) 1,8 millones de zeds aproximadamente

( ) 2,3 millones de zeds aproximadamente

( ) 3, 4 millones de zeds aproximadamente

( ) $\underline{3,8 \text { millones de zeds aproximadamente }}$ 
10. Se han considerado ocho personas para el puesto de Director Ejecutivo de una compañía. Seis de los candidatos tienen más de 60 años de edad. Tres son mujeres y de ellas dos tienen más de 60 años de edad. Si se selecciona un candidato aleatoriamente y resulta ser hombre, ¿cuál es la probabilidad de que tenga más de 60 años de edad?
( ) $3 / 5$
( ) $5 / 5$
( ) $\underline{4 / 5}$
( ) $5 / 4$

11. Considere que la distribución de dos grupos de datos toman las siguientes formas:

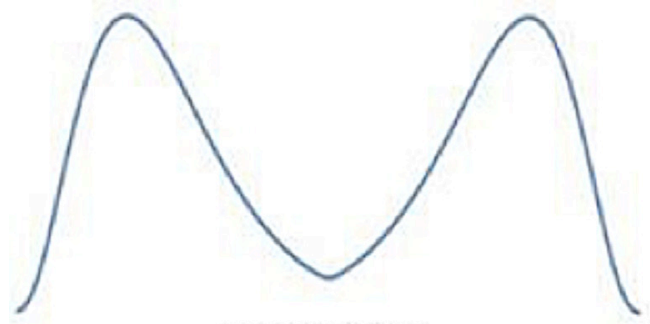

Distribución A

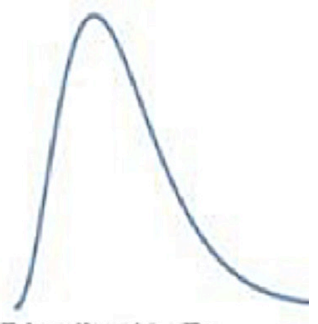

Distribucion B

Si se desea resumir por medio de una medida la posición en la que se encuentra la mayor concentración de datos, debería utilizar:

\section{( ) La moda en los dos casos}

( ) La mediana en el caso A y el promedio en el caso B

( ) La mediana en los dos casos

( ) El promedio en el caso A y la mediana en el caso B 
12. La opción que mejor caracteriza respectivamente a la variable sexo en términos del tipo de variable, medida de tendencia central y gráfica asociada es:

( ) Cualitativa, Media Aritmética, Circular

( ) Cuantitativa, Media Aritmética, Barras

( ) Cuantitativa, Mediana, Polígono de Frecuencias

( ) Cualitativa, Moda, Circular

13. La opción que mejor caracteriza respectivamente a la variable número de habitaciones en términos del tipo de variable, medida de tendencia central y gráfica asociada es:

( ) Cuantitativa Discreta, Mediana, Histograma

( ) Cuantitativa Continua, Media Aritmética, Diagrama de caja

( ) Cuantitativa Discreta, Media Aritmética, Barras

( ) Cuantitativa Continua, Moda, Histograma

14. La opción que mejor caracteriza respectivamente a la variable peso en kilogramos en términos del tipo de variable, medida de tendencia central y gráfica asociada es:

( ) Cuantitativa Continua, Moda, Circular

( ) Cuantitativa Continua, Mediana, Histograma

( ) Cuantitativa Discreta, Media Aritmética, Polígono de Frecuencia

( ) Cualitativa, Moda, Diagrama de caja 
Parte II: Desarrollo. Los siguientes ítems corresponden a desarrollo, por tanto debe presentar TODOS los pasos necesarios que le permitan obtener su respuesta.

15. Suponga que tenemos un circuito como el mostrado en la figura siguiente, en el cual se lanzan 100 bolitas por la parte superior:

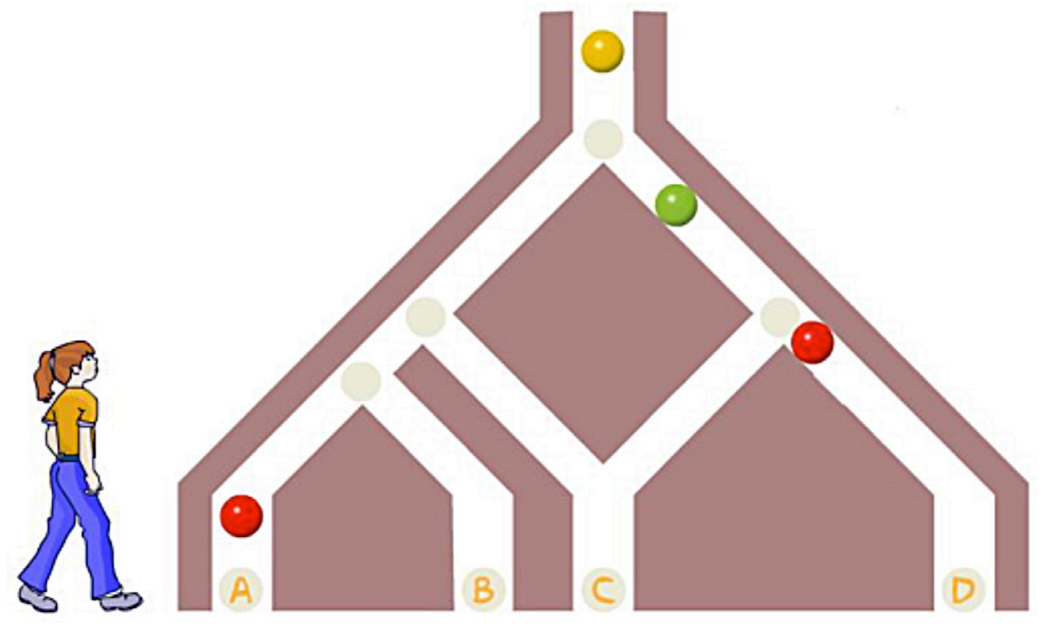

De acuerdo con la información anterior, ¿̇en cuál de los depósitos (A, B, $\underline{C}, \mathrm{D})$ caerán más bolitas? ¿Por qué?

16. Un juego de tiro al blanco consiste en dispararle a una sandía (que está lo suficientemente cerca como para acertar el tiro) con una pistola que dispara bolas de goma con pintura dentro. Para ello hay seis jugadores. El arma tiene un cargador giratorio para las balas y espacio para contener seis balas, pero solo se va a cargar con una bala. Cada jugador va a girar el cargador antes de disparar en su turno, sin conocer la posición de la bala; gana el jugador que logre dispararle a la sandía primero. ¿Considera que los seis participantes tienen igual posibilidad de dispararle exitosamente a la sandía?
( ) $\underline{\text { Sí }}$
( ) No

¿Por qué? 